\title{
Local Asymptotic Normality Complexity Arising in a Parametric Statistical Lévy Model
}

\author{
Wissem Jedidi \\ Department of Statistics and OR, King Saud University, P.O. Box 2455, Riyadh 11451, Saudi Arabia \\ Correspondence should be addressed to Wissem Jedidi; wissem_jedidi@yahoo.fr
}

Received 27 August 2021; Accepted 9 November 2021; Published 29 December 2021

Academic Editor: Ning Cai

Copyright (c) 2021 Wissem Jedidi. This is an open access article distributed under the Creative Commons Attribution License, which permits unrestricted use, distribution, and reproduction in any medium, provided the original work is properly cited.

We consider statistical experiments associated with a Lévy process $X=\left(X_{t}\right)_{t \geq 0}$ observed along a deterministic scheme $\left(i u_{n}, 1 \leq i \leq n\right)$. We assume that under a probability $\mathbb{P}_{\theta}$, the r.v. $X_{t}, t>0$, has a probability density function $>o$, which is regular enough relative to a parameter $\theta \in(0, \infty)$. We prove that the sequence of the associated statistical models has the LAN property at each $\theta$, and we investigate the case when $X$ is the product of an unknown parameter $\theta$ by another Lévy process $Y$ with known characteristics. We illustrate the last results by the case where $Y$ is attracted by a stable process.

\section{Introduction}

This work is a part of an ambitious program consisting in the estimation of the parameter $\theta$ intervening in the stochastic differential equation driven by a known Lévy process $Y$ :

$$
\mathrm{d} X_{t}=b(\theta, X) \mathrm{d} t+a(\theta, X) \mathrm{d} Y_{t},
$$

These kinds of models are motivated by mathematical finance problems ([1]). In this context, the property of local asymptotic normality property (LAN) has become an important issue [2]. The LAN property is described as follows: a sequence of families of probabilities $\left(\mathbb{P}_{\theta}^{n}\right)_{\theta \in \Theta}$ indexed by an open set $\Theta \subset \mathbb{R}$ is said to have the LAN property at each point $\theta_{0} \in \Theta$ with speed $\sqrt{n}$, if the sequence of probabilities localized around $\theta_{0}$,

$$
\left(\mathbb{P}_{\theta_{0}+n^{(-1 / 2)} \theta}^{n}\right)_{\theta \in\left\{\left(\xi /\left(\theta_{0}+n^{(-1 / 2)}\right)\right) \xi \in \Theta\right\}},
$$

converges, in the sense of weak convergence of the associated likelihood processes, to a Gaussian shift $\left(\mathbb{P}_{\theta^{\prime}}\right)_{\theta \in \mathbb{R}}$; see Section 2 for a precise definition. The LAN property allows to recover the so-called asymptotic Fisher information quantity $I\left(\theta_{0}\right)$. This quantity is crucial in any estimation procedure, since $1 / I\left(\theta_{0}\right)$ provides the lower bound of the variance of any estimator of $\theta_{0}$.
The LAN property was investigated by Akritas [3] in models associated with Lévy processes $X$ observed continuously in time over the interval $[0, n], n \longrightarrow \infty$. He obtained the property under the assumption of differentiability, according to the parameter $\theta$, of the characteristics $\left(b_{\theta}, c_{\theta}, v_{\theta}\right)$ of $X$. With the same asymptotic, Luschgy [4] obtained the local asymptotic mixed normality (LAMN) property on models associated with semimartingales. As a notion, LAMN property is more general than the LAN because it allows the Fisher information quantity to be random. With the asymptotic $[0, n], n \longrightarrow \infty$, the estimation methods do not seem to be feasible in practice, for this reason, several recent works focused on discretized schemes, i.e., observations of the process $X$ along the discrete scheme

$$
X_{i u_{n}}, \quad 1 \leq i \leq n, n \longrightarrow \infty .
$$

In practice, the most interesting case of the discretization path $u_{n}=1 / n$ turns out to be relatively difficult. The classical case of a Brownian motion $Y$ in (1) has been widely treated [5]. Clément and Gloter [6] studied the LAN for the model in (1), in the case where $Y$ is a Lévy process attracted by a symmetric stable process with index $\alpha \in(1,2]$. Aït-Sahalia and Jacod [7], Masuda [8], and Kawai and Masuda [9, 10] 
studied LAN property for the model in (1) in case of constant coefficients, i.e.,

$$
\mathrm{d} X_{t}=\theta_{1} \mathrm{~d} t+\theta_{2} \mathrm{~d} Y_{t},
$$

Our investigation goes to same direction of Ait-Sahalia and Jacod [11], who studied the LAN property and the problem of estimation of the parameter $\left(\theta_{1}, \theta_{2}\right)$ involved in the model of a log-asset price $X$, solution of (3) with $Y$ being a standard symmetric stable process with index $\alpha \in(0,2]$. Section 4 completes their situation in case where $Y$ is a general stable process, eventually mixed. The last direction was initiated Rammeh [12] with observations according to random schemes $(T(i, n), 1 \leq i \leq n)$ for the scale model:

$$
X=\theta Y \text {, }
$$

where $\theta$ is a real unknown real parameter, and $Y$ is a symmetrical standard $\alpha$-stable process. Rammeh showed that the LAN property always occurs, and his main arguments strongly rely to the linearity in $\theta$ to the fact that stable processes have the temporal scaling property and to the asymptotic behavior of the stable densities. Theorem 2 generalizes Rammeh's results in the context of deterministic discrete scheme $T(i, n)=i u_{n}$.

Because of the intricacy of the case (1), we first focus on the following model, which contains (5) and intercepts (1): we assume that for all $\theta \in \Theta$, under $\mathbb{P}_{\theta}, X$ is a Lévy process, null at $t=0$, such that its Lévy exponent is given by the socalled Lévy-Khintchine formula:

$$
\begin{aligned}
\mathbb{E}_{\mathbb{P}_{\theta}}\left[e^{i u X_{t}}\right]= & e^{t \varphi_{\theta}(u)}, \quad \varphi_{\theta}(u)=i u b_{\theta}-\frac{c_{\theta}^{2} u^{2}}{2} \\
& +\int_{\mathbb{R}}\left(e^{i u y}-1-i u y 1 l_{|y| \leq 1}\right) \mu_{\theta}(\mathrm{d} y),
\end{aligned}
$$

where $b_{\theta} \in \mathbb{R}, c_{\theta} \in \mathbb{R}_{+}$, and $\mu_{\theta}$ is a positive measure on $\mathbb{R}$ which integrates $\min \left(y^{2}, 1\right)$.

For sake of clarity, we take $\Theta$ is the open interval $\mathbb{R}$. As in the precited literature, we will assume the following.

(i) The existence of densities $g_{t}^{\theta}$, such that $\theta \mapsto g_{t}^{\theta}$ is regular enough,

(ii) The convergence, as $n \longrightarrow \infty$, of some integrals depending on $g_{u_{n}}^{\theta}$.

Theorem 1 and Corollary 1 provide conditions ensuring the LAN property for the model (6), when the process $X$ is observed along the discrete scheme (3). Denoting $\bar{g}_{u_{n}}^{\theta}$, the logarithmic derivative of $g_{u_{n}}^{\theta}$ relative to $\theta$, the asymptotic Fisher information quantity at each $\theta$ should satisfy

$$
I(\theta)=\lim _{n \longrightarrow \infty} \int\left(\bar{g}_{u_{n}}^{\theta}\right)^{2}(x) g_{u_{n}}^{\theta}(x) \mathrm{d} x \quad \in(0, \infty) .
$$

It is difficult to find Lévy processes fulfilling (7), and the reasons are numerous, for instance, the existence of the densities $g_{t}^{\theta}$, the fact that they are not explicit in general, and their degeneracy as $t \longrightarrow 0$. For these reasons, Corollary 1 focuses on the linear dependance (4) of the characteristics relative to $\theta$. In this case, we may assume, without loss of generality, that $\Theta$ contains a reference value, 1 for example, and the value 0 is excluded in order to avoid trivialities. In this case, we only need to assume some regularities of the function $g_{u_{n}}^{1}(x)$ and conditions of the kind (7) for $\theta=1$. Let $h_{n}:=g_{u_{n}}^{1}$ and $\bar{h}_{n}$ be the logarithmic derivatives of $h_{n}(x)$. The asymptotic Fisher information quantity should then satisfy

$$
I(\theta)=\frac{1}{\theta^{2}} \lim _{n \longrightarrow \infty} \int\left(1+x \bar{h}_{n}(x)\right)^{2} h_{n}(x) \mathrm{d} x \quad \in(0, \infty) .
$$

The case of the discretization with constant path $u_{n}=$ $u \in(0, \infty)$ is quite obvious since the scale model (5) becomes a regular i.i.d. one, that is, to say $I(\theta)$ is finite and nonnull. If $u_{n} \longrightarrow 0$, the situation is more intricate because $h_{n}$ degenerates when $n \longrightarrow \infty$. It turns out that even the linear model (5) is falsely simple to handle. Intuitively, one looks at special Lévy processes $Y$ attracted by stable processes on the sense of (10). The price to pay is to exhibit refined controls on the probability density function of $Y_{t}, t>0$. In a second step, we restrict our attention to the scale model (5). For simplicity's sake, it is easier in this case to express the probabilities $\left(\mathbb{P}_{\theta}\right)_{\theta \in \Theta}$ in the form (5) rather than considering them as solutions of martingale problems associated with the family of characteristics $\left(b_{\theta}, c_{\theta}, \mu_{\theta}\right)_{\theta \in \Theta}$ because of the intricacy inherent in the truncation functions [13]. Generic examples of Lévy processes are stable processes. They characterized Lévy exponent as follows. Let $(\alpha, \beta, \gamma, \delta) \in A=(0,2] \times[-1,1] \times$ $(0,+\infty) \times \mathbb{R}$ and

$$
\begin{aligned}
& K(\alpha)=\alpha 1 l_{(0,1]}(\alpha)+(\alpha-2) 1 l_{(1,2]}(\alpha), \\
& \bar{K}(\alpha)=\frac{K(\alpha)}{\alpha} .
\end{aligned}
$$

A stable process, with parameters $(\alpha, \beta, \gamma, \delta)$, is a Lévy process $X=\left(S_{t}^{\alpha, \beta, \gamma, \delta}\right)_{t \geq 0}$, such that the corresponding Lévy exponent is given by

$$
\varphi(u)= \begin{cases}i \delta u-\gamma|u|^{\alpha} \exp (-i \pi \beta K(\alpha) \operatorname{Sign}((u) / 2)), & \text { if } \alpha \neq 1, \\ i \delta u-\gamma|u|(1+i 2 \beta \log |u| \operatorname{Sign}(u) / \pi), & \text { if } \alpha=1 .\end{cases}
$$

The parameter $\alpha$ is the stability coefficient, $\beta$ is the skewness coefficient, $\gamma$ is the scale coefficient, and $\delta$ is the drift parameter. The corresponding triplet $(b, c, \mu)$ of characteristics is given by

$$
\begin{aligned}
b & =\delta+\gamma \sin (\pi \beta K((\alpha) / 2)) 1 l_{\alpha \neq 1}, \\
c & =\sqrt{2 \gamma} 1 l_{\alpha=2}, \\
\mu(\mathrm{d} x) & =\left(C(\alpha, \beta, \gamma) 1 l_{x>0}+C(\alpha,-\beta, \gamma) 1 l_{x<0}\right)|x|^{-(\alpha+1)} \mathrm{d} x 1 l_{\alpha<2}, \\
C(\alpha, \beta, \gamma) & =\frac{\gamma}{\pi} \Gamma(\alpha+1) \begin{cases}\sin \left(\frac{\pi}{2} \alpha(1+\bar{K}(\alpha) \beta)\right), & \text { if } \alpha \neq 1, \\
1+\beta, & \text { if } \alpha=1 .\end{cases}
\end{aligned}
$$

See [14]. In model (6), a candidate for the unknown parameter $\theta$ could be any the parameters $\alpha, \beta, \gamma$, or $\delta$. Since 
stable processes enjoy the scaling property, with $\delta(\alpha, t):=$ $t\left[\delta+(2 \beta / \pi) \gamma \log (\gamma) 1 l_{\alpha=1}\right]$,

$$
\left(S_{t}^{\alpha, \beta, \gamma, \delta}\right)_{t \geq 0} \stackrel{d}{=} \gamma^{1 / \alpha}\left(\delta(\alpha, t)+S_{t}^{\alpha, \beta, 1,0}\right)_{t \geq 0},
$$

then a candidate for the unknown parameter in the model (5) is clearly the parameter $\theta=\gamma^{1 / \alpha}$. For more account on Lévy processes, the reader is referred to [13] or [15] and for stable distributions, we suggest [16] and also [17]. Section 4 provides nontrivial examples of LAN models associated with Lévy processes $Y$ attracted by stable ones. That means that there exist measurable functions $b(t) \in \mathbb{R}, a(t)>0$ and a nondegenerate distribution $v$, such that

$$
\widehat{Y}_{t}=\frac{Y_{t}-b(t)}{a(t)} \stackrel{d}{\longrightarrow} \nu, \quad \text { as } t \longrightarrow 0 \text { or as } t \longrightarrow \infty .
$$

In [18], Far focused on the LAMN property for the model (5) discretized along the scheme $i u_{n}=(i / n), 1 \leq i \leq n$, when the process $Y$ is of the form $Y=W+N$, the sum of a standard Brownian motion and an independent compound Poisson process. She obtained LAMN property under the condition that the Lévy measure $v$ of $N$ has no diffuse singular part and that if $v$ is absolutely continuous, then the model has the LAN property. Our development in Section 5 constitutes a complement to Corollary 1 for the scale model (5) and also to Far's work [18] and illustrates how to build a LAN scale model from another LAN scale model.

\section{Definition of the LAN Property}

In Section 3, we provide some theoretical results on models associated with observations, at times $i u_{n}, u_{n}>0$, of the process $X$, and to illustrate by some examples. To this end, we consider the sequence of i.i.d. random variables and the family of $\sigma$-fields:

$$
\begin{aligned}
& X_{j}^{n}=X_{(j+1) u_{n}}-X_{j u_{n},} \\
& \mathscr{G}_{i}^{n}=\sigma\left(X_{j}^{n}, \quad 0 \leq j \leq i-1\right)=\sigma\left(j u_{n}, \quad 0 \leq j \leq i\right) .
\end{aligned}
$$

Denoting $\mathscr{H}^{n}=\mathscr{G}_{n}^{n}$ and $\mathscr{H}_{t}^{n}=\mathscr{G}_{[n t]}^{n}, t \in[0,1]$, we introduce the sequence of filtered statistical models:

$$
E^{n}=\left(\Omega, \mathscr{H}^{n},\left(\mathscr{H}_{t}^{n}\right)_{t \in[0,1]},\left(\mathbb{P}_{\theta}\right)_{\theta \in \Theta}\right) .
$$

For any fixed $\theta_{0} \in \Theta$, we denote

$$
\begin{aligned}
\Theta_{n} & =\left\{\theta \in \mathbb{R}: \quad \theta_{0}+\frac{\theta}{\sqrt{n}} \in \Theta\right\}, \\
{[\theta]_{n} } & =\theta_{0}+\frac{\theta}{\sqrt{n}}, \\
\mathbb{P}_{\theta}^{n} & =\mathbb{P}_{[\theta]_{n}} \mid \mathscr{H}^{n},
\end{aligned}
$$

and we introduce the statistical experiments localized around $\theta_{0}$ :

$$
\begin{aligned}
& \mathscr{E}^{n}\left(\theta_{0}\right)=\left(\Omega, \mathscr{H}^{n},\left(\mathscr{H}_{t}^{n}\right)_{t \in[0,1]},\left(\mathbb{P}_{\theta}^{n}\right)_{\theta \in \Theta_{n}}\right), \\
& \mathscr{E}^{\prime}\left(\theta_{0}\right)=\left(\Omega^{\prime}, \mathscr{F}^{\prime},\left(\mathscr{F}_{t}^{\prime}\right)_{t \in[0,1]},\left(\mathbb{P}_{\theta}^{\prime}\right)_{\theta \in \mathbb{R}}\right),
\end{aligned}
$$

where the last statistical experiment is a Gaussian Shift. By a Gaussian shift, we mean, that for all $\theta \in \mathbb{R}, \mathbb{P}_{\theta}^{\prime}$ is the unique probability on $\left(\Omega^{\prime}, \mathscr{F}^{\prime}\right)$ equivalent to $\mathbb{P}_{0}^{\prime}$ on each $\mathscr{F}_{t}^{\prime}$ and that its associated likelihood process is the geometric Brownian motion defined by

$$
Z_{t}^{\prime \theta}=\frac{\mathrm{d} \mathbb{P}_{\theta}^{\prime} \mid \mathscr{F}_{t}^{\prime}}{\mathrm{dP}_{\theta_{0}}^{\prime} \mid \mathscr{F}_{t}^{\prime}}=\exp \left\{\theta \sqrt{I\left(\theta_{0}\right)} X_{t}^{\prime}-\frac{\theta^{2}}{2} I\left(\theta_{0}\right) t\right\}, \quad t \in[0,1],
$$

where $\left(X_{t}^{\prime}\right)_{t \in[0,1]}$ is a Wiener process, and then, under $\mathbb{P}_{\theta}^{\prime}$, the process $\left(X_{t}^{\prime}-t \theta \sqrt{I\left(\theta_{0}\right)}\right)_{t \geq 0}$ is again a Wiener process. The quantity $I\left(\theta_{0}\right)$ is called the asymptotic Fisher information quantity; it is a positive constant related to the sequence of statistical experiments $\mathscr{E}^{n}\left(\theta_{0}\right)$ in (17) and has to be determined. The asymptotic Fisher information quantity is crucial in any estimation procedure. Indeed, under the LAN property, $1 / I\left(\theta_{0}\right)$ is the lower bound of the variance of any estimator $\vartheta_{n}$ of $\theta_{0}$. More precisely, HAJEK's asymptotic convolution theorem says that if $\vartheta_{n}$ satisfies

$$
\mathscr{L} a w\left(\sqrt{n}\left(\vartheta_{n}-\left(\theta_{0}+\frac{\theta}{\sqrt{n}}\right)\right) \mid \mathbb{P}_{\theta}^{n}\right) \longrightarrow \mathscr{L}_{\theta_{0}}, \quad \text { as } n \longrightarrow \infty,
$$

then the distribution $\mathscr{L}_{\theta_{0}}$ is the convolution product $\mathscr{L}_{\theta_{0}}=\mathscr{L}_{\theta_{0}}^{1} * \mathscr{L}_{\theta_{0}}^{2}$, where $\mathscr{L}_{\theta_{0}}^{1}=\operatorname{normal}\left(0, I\left(\theta_{0}\right)^{-1}\right)$ and $\mathscr{L}_{\theta_{0}}^{2}$ is a probability measure on $\mathbb{R}$. See [19] for more.

Local asymptotic normality of the sequence of models $E^{n}$ in (17), in a value $\theta_{0} \in \Theta$, is actually equivalent to the weak functional convergence in time of the sequence of statistical experiments $\mathscr{E}^{n}\left(\theta_{0}\right)$ to the Gaussian shift $\mathscr{E}^{\prime}\left(\theta_{0}\right)$ in (17). This fact is explained as follows: let $Z^{\prime \eta \xi}$ and $Z^{n, \eta \xi}$ be the likelihood processes defined, for all $\eta, \xi \in \Theta_{n}$ and at each time $t \in[0,1]$, by

$$
\begin{aligned}
Z_{t}^{\prime} \eta \xi & =\frac{\mathrm{d} \mathbb{P}_{\eta}^{\prime} \mid \mathscr{F}_{t}^{\prime}}{\mathrm{d} \mathbb{P}_{\xi}^{\prime} \mid \mathscr{F}_{t}^{\prime}}=\mathbb{E}_{\mathbb{P}_{\xi}^{\prime}}\left[\frac{\mathrm{d} \mathbb{P}_{\eta}^{\prime}}{\mathrm{d} \mathbb{P}_{\xi}^{\prime}} \mid \mathscr{F}_{t}^{\prime}\right]=\frac{Z_{t}^{\prime \eta}}{Z_{t}^{\prime \xi},} \\
Z_{t}^{n, \eta \xi} & =\frac{\mathrm{d} \mathbb{P}_{\eta}^{n} \mid \mathscr{H}_{t}^{n}}{\mathrm{~d} \mathbb{P}_{\xi}^{n} \mid \mathscr{H}_{t}^{n}}=\mathbb{E}_{\mathbb{P}_{\xi}^{n}}\left[\frac{\mathrm{d} \mathbb{P}_{\eta}^{n}}{\mathrm{~d} \mathbb{P}_{\xi}^{n}} \mid \mathscr{H}_{t}^{n}\right],
\end{aligned}
$$

with the convention $(a / 0)=0$, if $a \in(0,+\infty)$. According to [5], the likelihood process $Z^{n, \eta \xi}$ of the statistical experiment $\mathscr{E}^{n}\left(\theta_{0}\right)$ is represented by

$$
Z_{t}^{n, \eta \xi}=\prod_{j=1}^{[n t]} \frac{g_{u_{n}}^{\eta}}{g_{u_{n}}^{\xi}}\left(X_{j}^{n}\right)
$$

The notion of weak functional convergence in time was introduced by Lecam [2] and developed by Strasser [19] and Jacod [20]. It is expressed as follows: for every finite subset $J$ of $\mathbb{R}=\cup_{n \geq 1} \Theta_{n}$, and every $\xi \in \Theta$, we have

$$
\begin{aligned}
& \mathscr{L} a w\left(\left(Z^{n, \eta \xi}\right)_{\eta \in J} \mid \mathbb{P}_{[\xi]_{n}}^{n}\right) \longrightarrow \mathscr{L} a w\left(\left(Z^{\prime \eta \xi}\right)_{\eta \in J} \mid \mathbb{P}_{\xi}^{\prime}\right), \\
& \text { as } n \longrightarrow+\infty \text {, }
\end{aligned}
$$


in the sense of the weak convergence for the Skorohod topology.

\section{When Does LAN Property Hold for Lévy Models?}

Our aim is to give sufficient conditions on the p.d.f. $>o$ of $X_{t}$ under $\mathbb{P}_{\theta}$, ensuring the LAN property for the sequence of filtered statistical models $E^{n}$.
3.1. LAN Property for the Model (6). In this section, we will consider the model (6). If $c_{\theta}=0, \mu_{\theta}$ integrates $|y| \wedge 1$, and $\mu_{\theta}(0, \infty)=0$ (respectively, $\left.\mu_{\theta}(-\infty, 0)=0\right)$, then the support of the distribution of $X_{t}$ is

$$
\left[d_{\theta} t, \infty\left(\text { respectively, }\left(-\infty, d_{\theta} t\right]\right) \text {, with } d_{\theta}=b_{\theta}-\int_{\mathbb{R}} y 1 l_{|y| \leq 1} \mu_{\theta}(\mathrm{d} y)\right. \text {. }
$$

In all other cases, the distribution of $X_{t}$ has a support equal to $\mathbb{R}$. There are many situations in which for all $t>0, X_{t}$ has a probability p.d.f. $g_{t}^{\theta}(x)$ which is infinitely differentiable in $x$. For instance, the latter holds if

$$
\begin{aligned}
c_{\theta} & >0, \\
\text { or if } \int_{|y| \leq \varepsilon} \min \left(y^{2}, 1\right) \mu_{\theta}(\mathrm{d} y) & \geq K_{\theta} \varepsilon^{\alpha},
\end{aligned}
$$

for any $\varepsilon \in[0,1]$ and for some $K_{\theta}>0$ and some $\alpha \in(0,2)$, see [21]. Later on, we may assume the following:

(H0): for all $\theta \in \Theta$ and $t>0$, under $\mathbb{P}_{\theta}$, the support of the law of $X_{t}$ is an interval $K_{t}$,

independent from $\theta$, of the form $K_{t}=\mathbb{R}$ or $(-\infty, \mathrm{d} t$

and $X_{t}$ has a p.d.f. $x \mapsto g_{t}^{\theta}(x)$ which is of class $C^{2}$, relative to $\theta$.

We denote $\mathscr{K}_{n}=K_{u_{n}}$ and we define, on the interior of $\mathscr{K}_{n}$, the following functions:

$$
\begin{aligned}
& h_{n}^{\theta}=g_{u_{n}}^{\theta}, \\
& \bar{h}_{n}^{\theta}=\frac{\partial}{\partial_{\theta}} \log h_{n}^{\theta}, \\
& \ddot{h}_{n}^{\theta}=\frac{\partial^{2}}{\partial_{\theta}^{2}} h_{n}^{\theta}, \\
& i_{n}^{\theta}=h_{n}^{\theta}\left|\bar{h}_{n}^{\theta}\right|^{2} \\
& j_{n}^{\theta}=i_{n}^{\theta}+\left|\ddot{h}_{n}^{\theta}\right| .
\end{aligned}
$$

When the number $\chi>0$ appears, it is always understood that $n$ is big enough so that $\chi$ and $-\chi$ are in $\Theta_{n}$. For all $\theta \in \Theta, \rho \in(0,1)$, and $\rho^{\prime}=1-\rho$, we denote

$$
\begin{aligned}
I_{n}^{\theta} & :=\int_{\mathscr{K}_{n}} i_{n}^{\theta}(x) \mathrm{d} x, \\
\widetilde{I}_{n}(\chi) & =\sup _{|\varepsilon| \leq \chi} I_{n}^{[\varepsilon]_{n}}, \\
\widetilde{J}_{n}^{\rho}(\chi) & :=\sup _{|\zeta||| \varepsilon \mid \leq \chi} \int_{\mathscr{K}_{n}} \frac{j_{n}^{[\zeta]_{n}}(x) j_{n}^{[\varepsilon]_{n}}(x)}{\left(h_{n}^{[\zeta]_{n}}(x)\right)^{\rho}\left(h_{n}^{[\varepsilon]_{n}}(x)\right)^{\rho^{\prime}}} \mathrm{d} x=\widetilde{J}_{n}^{\rho^{\prime}}(\chi) .
\end{aligned}
$$

For statisticians, $I_{n}^{\theta_{0}}$ is a familiar quantity and corresponds to the Fisher information quantity at stage $n$. The quantity $\widetilde{J}_{n}^{\rho}(\chi)$ is less intuitive; it is a localized quantity around the true value $\theta_{0}$ and corresponds to the rest of Taylor approximations at the order 1 of Hellinger integrals of the model.

We are now able to state our first result, that is, the LAN property for the model (6).

Theorem 1. Assume (HO) and the following conditions: 
(H1): $\lim _{n \longrightarrow \infty} I_{n}^{\theta_{0}}=I\left(\theta_{0}\right) \in(0, \infty)$ and for all $\chi>0, \limsup _{n \longrightarrow+\infty} \widetilde{I}_{n}(\chi)<+\infty$,

(H2): There exists $a \in(0,(1 / 2))$ such that for all $\chi>0$, one has

$$
\lim _{n \longrightarrow+\infty} \frac{\tilde{J}_{n}^{\rho}(\chi)}{n}=0 \quad \text { for } \rho \in\{(1 / 2), a, 1-a\} .
$$

Then, the sequence of sequence of filtered statistical models $E^{n}$ (15), corresponding to (6), has the LAN property at $\theta_{0}$ with the speed $\sqrt{n}$ and the asymptotic Fisher information quantity $I\left(\theta_{0}\right)$.

\section{Remark 1}

(i) Cauchy-Schwarz inequality gives $I_{n}^{\theta} \leq\left[\widetilde{J}_{n}^{1 / 2}(|\theta|]\right]^{1 / 2}$, and both conditions (H1) and (H2) are implied by

$$
\begin{aligned}
& \text { (H3): } \lim _{n \longrightarrow+\infty} I_{n}^{\theta_{0}}=I\left(\theta_{0}\right) \text { and there exists } a \in(0,(1 / 2)) \text {, such that for all } \chi>0 \text {, one has } \\
& \quad \limsup _{n \longrightarrow+\infty}^{\rho} \widetilde{J}_{n}^{\rho}(\chi)<+\infty \quad \text { for } \rho \in\{(1 / 2), a, 1-a\} .
\end{aligned}
$$

(ii) Under different conditions and a different proof, Masuda obtained ([8], Theorem 2.12) the same conclusion as in Theorem 1.

Genon-Catalot and Jacod [5] exhibited discretized models according to random sampling schemes $(T(i, n), 1 \leq i \leq n)$ associated with a diffusion process $X$ driven by Brownian motions (with coefficients dependent on $\theta$ and by an homogeneous way on $X$ ) and proved the LAMN property under conditions similar to (H0), that is, differentiability to the third order relative to $\theta$ and integrability of the densities of the processes. Their proofs have a general vocation in the sense that they only use the Markovian property of the processes and are based on a method of approximation of the log-likelihood. Because of the intricate form 16 of the likelihood processes, we show the weak functional convergence of $\mathscr{E}^{n}\left(\theta_{0}\right)$ to $\mathscr{E}^{\prime}\left(\theta_{0}\right)$ via the convergence of the Hellinger processes, and with a tool, one can find in $[20]$.

Proof of Theorem 1. Fix $\theta_{0}$. The Hellinger process of order $\rho \in(0,1)$ between $\mathbb{P}_{\eta}^{\prime}$ and $\mathbb{P}_{\xi}^{\prime}$, relative to $\left(\mathscr{F}_{t}^{\prime}\right)_{t \in[0,1]}$, is deterministic and has the form

$$
\mathscr{H}^{\prime \eta \xi}(\rho)_{t}=\rho(1-\rho)(\eta-\xi)^{2} I\left(\theta_{0}\right)\left(\frac{t}{2}\right) .
$$

According to ([20], Theorem 5.3), it is enough to show that the Hellinger processes $\mathscr{H}^{n, \eta \xi}(\rho)$ between $\mathbb{P}_{\eta}^{n}$ and $\mathbb{P}_{\xi}^{n}$, relative to $\left(\mathscr{G}_{t}^{n}\right)_{t \in[0,1]}$, satisfy the following: there exists $a \in(0,(1 / 2))$, such that for every $\forall \eta, \xi \in \mathbb{R}$, $\rho \in\{(1 / 2), a, 1-a\}$, and $t \in[0,1]$, and the convergence in law

$$
\mathscr{H}^{n, \eta \xi}(\rho)_{t} \stackrel{\mathbb{P}_{\xi}^{n}}{\longrightarrow} \mathscr{H}^{\eta^{\eta \xi}}(\rho)_{t}, \quad \text { as } n \longrightarrow \infty,
$$

holds. We will use this method because in our framework, the processes $\mathscr{H}^{n, \eta \xi}$ are also deterministic and have the following quite simple form one can find in [5]: with $\lfloor n t\rfloor$ being the integer part of $n t$, we have

$$
\mathscr{H}^{n, \eta \xi}(\rho)_{t}=\lfloor n t\rfloor\left(1-\int_{\mathscr{K}_{n}}\left(h_{n}^{[\eta]_{n}}\right)^{\rho}\left(h_{n}^{[\xi]_{n}}\right)^{1-\rho}(y) \mathrm{d} y\right) .
$$

(1) For $\rho \in(0,1), \rho^{\prime}=1-\rho$, take $\Phi_{\rho}(u, v)=\rho u+\rho^{\prime} v-$ $u^{\rho} v^{\rho^{\prime}}, u, v \geq 0$, and observe that

$$
\mathscr{H}^{n, \eta \xi}(\rho)_{1}=\int_{\mathscr{K}_{n}} \Phi_{\rho}\left(h_{n}^{[\eta]_{n}}, h_{n}^{[\xi]_{n}}\right)(y) \mathrm{d} y .
$$

According to (32) and (33), it is enough to show that $\forall \eta, \xi \in \mathbb{R}$, and $\rho \in\{(1 / 2), a, 1-a\}$, and we have

$$
\lim _{n \longrightarrow \infty} n \int_{\mathscr{K}_{n}} \Phi_{\rho}\left(h_{n}^{[\eta]_{n}}, h_{n}^{[\xi]_{n}}\right)(y) \mathrm{d} y=\frac{\rho \rho^{\prime}}{2}(\eta-\xi)^{2} I\left(\theta_{0}\right) .
$$

(2) Assume (H0), (H1), and (H2) for a fixed $a \in(0,(1 / 2))$. Applying Taylor expansion at the first order of $\theta \mapsto\left(h_{n}^{\theta}\right)^{\rho}$ for $\eta \in \mathbb{R}$ and $n$ big enough, we get for $\eta \in \Theta_{n}$, the representation of $\left(h_{n}^{[\eta]_{n}}\right)^{\rho}$ on $\mathscr{K}_{n}$ :

$$
\left(h_{n}^{[\eta]_{n}}\right)^{\rho}=\left(h_{n}^{\theta_{0}}\right)^{\rho}+\frac{\rho \eta}{\sqrt{n}} k_{n}^{\theta_{0}, \rho}+\frac{\rho \eta}{\sqrt{n}} V_{n}^{\eta, \rho},
$$

where for all $\theta \in \Theta$, the functions

$$
\begin{aligned}
k_{n}^{\theta, \rho} & =\bar{h}_{n}^{\theta}\left(h_{n}^{\theta}\right)^{\rho}, \dot{k}_{n}^{\theta, \rho}=\left(h_{n}^{\theta}\right)^{\rho}\left[\frac{\partial}{\partial \theta} \bar{h}_{n}^{\theta}+\rho\left(\bar{h}_{n}^{\theta}\right)^{2}\right] \\
& =\left(h_{n}^{\theta}\right)^{\rho}\left[\frac{\ddot{h}_{n}^{\theta}}{h_{n}^{\theta}}-\rho^{\prime}\left(\bar{h}_{n}^{\theta}\right)^{2}\right], \\
V_{n}^{\eta, \rho} & =\int_{0}^{1}\left[k_{n}^{[\eta r]_{n}, \rho}-k_{n}^{\theta, \rho}\right] \mathrm{d} r=\frac{\eta}{\sqrt{n}} \int_{0}^{1}(1-r) \dot{k}_{n}^{[\eta r]_{n}, \rho} \mathrm{d} r,
\end{aligned}
$$


are defined on the interior of $\mathscr{K}_{n}$. Also, observe the following Because of (38) and (39), one has relations:

$$
\begin{aligned}
k_{n}^{\theta, \rho}\left(h_{n}^{\theta}\right)^{\rho^{\prime}} & =k_{n}^{\theta, \rho^{\prime}}\left(h_{n}^{\theta}\right)^{\rho}=k_{n}^{\theta, 1}, \\
k_{n}^{\theta, \rho} k_{n}^{\theta, \rho^{\prime}} & =i_{n}^{\theta}, \\
\dot{k}_{n}^{\theta, \rho}\left(h_{n}^{\theta}\right)^{\rho^{\prime}} & =\dot{k}_{n}^{\theta, 1}-\rho^{\prime} i_{n}^{\theta} .
\end{aligned}
$$

$$
\begin{aligned}
V_{n}^{\eta, 1}-\left(h_{n}^{\theta_{0}}\right)^{\rho^{\prime}} V_{n}^{\eta, \rho} & =\frac{\eta}{\sqrt{n}} \int_{0}^{1}(1-r)\left[\dot{k}_{n}^{[\eta r]_{n}, 1}-\left(h_{n}^{\theta_{0}}\right)^{\rho^{\prime}} \dot{k}_{n}^{[\eta r]_{n}, \rho}\right] \mathrm{d} r \\
& =\frac{\eta}{\sqrt{n}} \int_{0}^{1}(1-r) \dot{k}_{n}^{[\eta r]_{n}, \rho}\left[\left(h_{n}^{[\eta r]_{n}}\right)^{\rho^{\prime}}-\left(h_{n}^{\theta_{0}}\right)^{\rho^{\prime}}\right] \mathrm{d} r+\frac{\eta \rho^{\prime}}{\sqrt{n}} \int_{0}^{1}(1-r) i_{n}^{[\eta r]_{n}} \mathrm{~d} r .
\end{aligned}
$$

Using (36), finally write

$$
V_{n}^{\eta, 1}-\left(h_{n}^{\theta_{0}}\right)^{\rho^{\prime}} V_{n}^{\eta, \rho}=\frac{\eta^{2} \rho^{\prime}}{n} \int_{0}^{1}(1-r) \dot{k}_{n}^{[\eta r]_{n}, \rho}\left[k_{n}^{\theta_{0}, \rho^{\prime}}+V_{n}^{\eta r, \rho^{\prime}}\right] \mathrm{d} r+\frac{\eta \rho^{\prime}}{\sqrt{n}} \int_{0}^{1}(1-r) i_{n}^{[\eta r]_{n}} \mathrm{~d} r
$$

(3) For all $\eta, \xi \in \mathbb{R}$ and $n$ large enough, so that $\eta$, $\xi \in \Theta_{n}$, write

$$
\begin{aligned}
\Phi_{\rho}\left(h_{n}^{[\eta]_{n}}, h_{n}^{[\xi]_{n}}\right)= & \rho\left[h_{n}^{\theta_{0}}+\frac{\eta}{\sqrt{n}} k_{n}^{\theta_{0}, 1}+\frac{\eta}{\sqrt{n}} V_{n}^{\eta, 1}\right]+\rho^{\prime}\left[h_{n}^{\theta_{0}}+\frac{\xi}{\sqrt{n}} k_{n}^{\theta_{0}, 1}+\frac{\xi}{\sqrt{n}} V_{n}^{\xi, 1}\right] \\
& -\left[\left(h_{n}^{\theta_{0}}\right)^{\rho}+\frac{\rho \eta}{\sqrt{n}} k_{n}^{\theta_{0}, \rho}+\frac{\rho \eta}{\sqrt{n}} V_{n}^{\eta, \rho}\right]\left[\left(h_{n}^{\theta_{0}}\right)^{\rho^{\prime}}+\frac{\rho^{\prime} \xi}{\sqrt{n}} k_{n}^{\theta_{0}, \rho^{\prime}}+\frac{\rho^{\prime} \xi}{\sqrt{n}} V_{n}^{\xi, \rho^{\prime}}\right] .
\end{aligned}
$$

Then, use (39) and obtain

$$
\begin{aligned}
n \Phi_{\rho}\left(h_{n}^{[\eta]_{n}}, h_{n}^{[\xi]_{n}}\right)= & \sqrt{n} \rho \eta\left[V_{n}^{\eta, 1}-\left(h_{n}^{\theta_{0}}\right)^{\rho^{\prime}} V_{n}^{\eta, \rho}\right]+\sqrt{n} \rho^{\prime} \xi\left[V_{n}^{\xi, 1}-\left(h_{n}^{\theta_{0}}\right)^{\rho} V_{n}^{\eta, \rho^{\prime}}\right] \\
& -\rho \rho^{\prime} \eta \xi\left[i_{n}^{\theta_{0}}+V_{n}^{\eta, \rho} V_{n}^{\xi, \rho^{\prime}}+k_{n}^{\theta_{0}, \rho} V_{n}^{\xi, \rho^{\prime}}+k_{n}^{\theta_{0}, \rho^{\prime}} V_{n}^{\eta, \rho}\right] .
\end{aligned}
$$

According to (41), we have

$$
n \Phi_{\rho}\left(h_{n}^{[\eta]_{n}}, h_{n}^{[\xi]_{n}}\right)=\rho \rho^{\prime}\left[A_{n}^{\eta, \xi}+B_{n}^{\eta, \xi}\right]
$$

$$
\begin{aligned}
A_{n}^{\eta, \xi}= & \eta^{2} \int_{0}^{1}(1-r) i_{n}^{[\eta r]_{n}} \mathrm{~d} r+\xi^{2} \int_{0}^{1}(1-r) i_{n}^{[\xi r]_{n}} \mathrm{~d} r-\eta \xi \dot{i}_{n}^{\theta_{0}}, \\
B_{n}^{\eta, \xi}= & \frac{\eta^{3}}{\sqrt{n}} \int_{0}^{1}(1-r) \dot{k}_{n}^{[\eta r r]_{n}, \rho}\left[k_{n}^{\theta_{0}, \rho^{\prime}}+V_{n}^{\eta r, \rho^{\prime}}\right] \mathrm{d} r+\frac{\xi^{3}}{\sqrt{n}} \int_{0}^{1}(1-r) \dot{k}_{n}^{[\xi r]_{n}, \rho^{\prime}}\left[k_{n}^{\theta_{0}, \rho}+V_{n}^{\xi r, \rho}\right] \mathrm{d} r, \\
& -\eta \xi\left[k_{n}^{\theta_{0}, \rho} V_{n}^{\xi, \rho^{\prime}}+k_{n}^{\theta_{0}, \rho^{\prime}} V_{n}^{\eta, \rho}+V_{n}^{\eta, \rho} V_{n}^{\xi, \rho^{\prime}}\right] .
\end{aligned}
$$


(4a) We will now prove the following convergence that will imply (35): for all $\eta, \xi \in \mathbb{R}$,

$$
\begin{aligned}
& \lim _{n \longrightarrow \infty} \int_{\mathscr{K}_{n}} A_{n}^{\eta, \xi}(x) \mathrm{d} x=\frac{1}{2}(\eta-\xi)^{2} I\left(\theta_{0}\right), \\
& \lim _{n \longrightarrow \infty} \int_{\mathscr{K}_{n}} B_{n}^{\eta, \xi}(x) \mathrm{d} x=0 .
\end{aligned}
$$

(4b). To prove (46), we use both (26) and (37), and for all $\rho \in(0,1)$ and $\theta \in \Theta$, we have the representation and the control.

$$
\begin{aligned}
& \left|k_{n}^{\theta, \rho}\right|=\sqrt{i_{n}^{\theta}}\left(h_{n}^{\theta}\right)^{\rho-(1 / 2)}, \\
& \left|\dot{k}_{n}^{\theta, \rho}\right| \leq \frac{j_{n}^{\theta}}{\left(h_{n}^{\theta}\right)^{\rho^{\prime}}}
\end{aligned}
$$

Let $\eta, \xi \in \mathbb{R}$, and let $\chi=|\eta| \vee|\xi|$. Using (26), (37), and Taylor expansion at the first order of $\theta \mapsto\left(k_{n}^{\theta,(1 / 2)}\right)^{2}$, we obtain the galloping control, valid for all $r \in[0,1]$ :

$$
\begin{aligned}
I_{n}^{[\eta r]_{n}}-I_{n}^{\theta_{0}} & =\int_{\mathscr{K}_{n}}\left[k_{n}^{[\eta r]_{n},(1 / 2)}(x)^{2}-k_{n}^{\theta_{0},(1 / 2)}(x)^{2}\right] \mathrm{d} x \\
& =\frac{2 \eta r}{\sqrt{n}} \iint_{0}^{1} k_{n}^{[\eta r s]_{n},(1 / 2)}(x) \dot{k}_{n}^{[\eta r s]_{n},(1 / 2)}(x) \mathrm{d} s \mathrm{~d} x .
\end{aligned}
$$

Taking $\rho=1 / 2$ in (48), applying Cauchy-Schwarz inequality and assuming (H1) and (H2), we obtain

$$
\sup _{r \in[0,1]}\left|I_{n}^{[\eta r]_{n}}-I_{n}^{\theta_{0}}\right| \leq 2|\eta| \widetilde{I}_{n}(\chi)\left(\frac{\widetilde{J}_{n}^{1 / 2}(\chi)}{n}\right)^{1 / 2} \longrightarrow 0 \quad \text { as } n \longrightarrow \infty \text {. }
$$

We then can write

$$
\begin{aligned}
\int_{\mathscr{K}_{n}} A_{n}^{\eta, \xi}(x) \mathrm{d} x & =\eta^{2} \int_{0}^{1}(1-r) I_{n}^{[\eta r]_{n}} \mathrm{~d} r+\xi^{2} \int_{0}^{1}(1-r) I_{n}^{[\xi r]_{n}} \mathrm{~d} r-\eta \xi I_{n}^{\theta_{0}} \\
& =\eta^{2} \int_{0}^{1}(1-r)\left(I_{n}^{[\eta r]_{n}}-I_{n}^{\theta_{0}}\right) \mathrm{d} r+\xi^{2} \int_{0}^{1}(1-r)\left(I_{n}^{[\xi r]_{n}}-I_{n}^{\theta_{0}}\right) \mathrm{d} r+\frac{1}{2}\left(\eta^{2}+\xi^{2}-2 \eta \xi\right) I_{n}^{\theta_{0}}
\end{aligned}
$$

and clearly (50) implies (46).

(4c). To prove (47), we use same the same arguments as in (4b), the Taylor expansion at the first order of $\theta \mapsto k_{n}^{\theta,(1 / 2)}$, and the representation

$$
\begin{array}{r}
\dot{k}_{n}^{[\eta r]_{n}, \rho} k_{n}^{\theta_{0}, \rho^{\prime}}=\dot{k}_{n}^{[\eta r]_{n}, \rho} k_{n}^{[\eta r]_{n}, \rho^{\prime}}-\frac{\eta r}{\sqrt{n}} \int_{0}^{1} \dot{k}_{n}^{[\eta r]_{n}, \rho} \dot{k}_{n}^{[\eta r s]_{n}, \rho^{\prime}} \mathrm{d} s, \\
\rho \in(0,1), r \in[0,1] .
\end{array}
$$

By (48) and Cauchy-Schwarz inequality, we have the following control, valid for all $r \in[0,1]$ :

$$
\begin{aligned}
& \int_{\mathscr{K}_{n}}\left|\dot{k}_{n}^{[\eta r]_{n}, \rho}(x) k_{n}^{\theta_{0}, \rho^{\prime}}(x)\right| \mathrm{d} x \\
& \leq \int_{\mathscr{K}_{n}} \sqrt{i_{n}^{[\eta r]_{n}}(x)} \frac{j_{n}^{[\eta r]_{n}}(x)}{\sqrt{h_{n}^{[\eta r]_{n}}(x)}} \mathrm{d} x+\frac{|\eta| r}{\sqrt{n}} \widetilde{J}_{n}^{\rho}(\chi) .
\end{aligned}
$$

$$
\int_{\mathscr{K}_{n}}\left|\dot{k}_{n}^{[\eta r]_{n}, \rho}(x) V_{n}^{\eta r, \rho^{\prime}}(x)\right| \mathrm{d} x \leq \frac{|\eta| r}{\sqrt{n}} \int_{0}^{1}(1-s) \int_{\mathscr{K}_{n}}\left|\dot{k}_{n}^{[\eta r]_{n}, \rho}(x) \dot{k}_{n}^{[\eta r s]_{n}, \rho^{\prime}}(x)\right| \mathrm{d} x \mathrm{~d} s,
$$

and reproducing the method, we used to get (54), and we obtain

$$
\sup _{r \in[0,1]} \int_{\mathscr{K}_{n}}\left|\dot{k}_{n}^{[\eta r]_{n}, \rho}(x) V_{n}^{\eta r, \rho^{\prime}}(x)\right| \mathrm{d} x \leq \delta_{n}^{\rho}(\chi) .
$$


According to (38) and (54), we also have

$$
\int_{\mathscr{K}_{n}}\left|k_{n}^{\theta_{0}, \rho}(x) V_{n}^{\eta, \rho^{\prime}}(x)\right| \mathrm{d} x \leq \frac{|\eta|}{\sqrt{n}} \int_{0}^{1}(1-r) \int_{\mathscr{K}_{n}}\left|\dot{k}_{n}^{[\eta r]_{n}, \rho^{\prime}}(x) k_{n}^{\theta_{0}, \rho}(x)\right| \mathrm{d} x \mathrm{~d} r \leq \frac{\chi}{\sqrt{n}} \delta_{n}^{\rho^{\prime}}(\chi) .
$$

Furthermore, (38), (48), and Cauchy-Schwarz inequality imply

$$
\int_{\mathscr{K}_{n}}\left|V_{n}^{\eta, \rho}(x) V_{n}^{\xi, \rho^{\prime}}(x)\right| \mathrm{d} x \leq \frac{|\eta \xi|}{n} \int_{0}^{1} \int_{0}^{1}(1-r)(1-s) \int \frac{j_{n}^{[\eta r]_{n}}(x) j_{n}^{[\xi s]_{n}}(x)}{h_{n}^{[\eta r]_{n}}(x)^{\rho^{\prime}} h_{n}^{[\xi s]_{n}}(x)^{\rho}} \mathrm{d} x \mathrm{~d} s \mathrm{~d} r \leq \frac{|\chi|}{\sqrt{n}} \delta_{n}^{\rho^{\prime}}(\chi) .
$$

Finally, according to (45), (54), (56)-(58), we obtain the control

$$
\int_{\mathscr{K}_{n}} B_{n}^{\eta, \xi}(x) \mathrm{d} x \leq 7 \chi^{3} \frac{\delta_{n}^{\rho}(\chi)}{\sqrt{n}}=7 \chi^{3}\left[\left(\widetilde{I}_{n}(\chi) \frac{\widetilde{J}_{n}^{(1 / 2)}(\chi)}{n}\right)^{(1 / 2)}+\chi \frac{\widetilde{J}_{n}^{\rho}(\chi)}{n}\right],
$$

and we conclude with the fact that assumptions (H1) and (H2) imply (47).

3.2. LAN Property for the Scale Model (5). As a consequence of Theorem 1, we obtain a result for the scale model (5). The main argument is that the r.v. $X_{t}, t>0$ has a p.d.f $g_{t}^{1}$ under $\mathbb{P}_{1}$ if and only if $X_{t}$ has the p.d.f. $g_{t}^{\theta}(x)=g_{t}^{1}(x / \theta) / \theta$ under $\mathbb{P}_{\theta}$. From now on, the functions $h_{n}, h_{n}^{\prime}$, and $h_{n}^{\prime \prime}$ denote, respectively, $h_{n}^{1}$, the p.d.f. of $X_{u_{n}}$ under $\mathbb{P}_{1}$, the first and the second derivatives of $h_{n}$. For $\theta \in \Theta$ and $n \geq 1$, we have the expression

$$
h_{n}^{\theta}(x)=\frac{1}{\theta} h_{n}\left(\frac{x}{\theta}\right), \quad \frac{x}{\theta} \in \mathscr{K}_{n}:=\operatorname{support}\left(h_{n}\right) .
$$

Therefore, if we want (H0) to be satisfied, we need to impose $K=\mathscr{K}_{n}=\mathbb{R}$ or $\mathbb{R}_{+}$or $\mathbb{R}_{-}$. Then, for all $x \in K$, we have

$$
\begin{aligned}
& i_{n}^{\theta}(x)=\frac{1}{\theta^{3}} i_{n}\left(\frac{x}{\theta}\right), \\
& j_{n}^{\theta}(x)=\frac{1}{\theta^{3}} j_{n}\left(\frac{x}{\theta}\right),
\end{aligned}
$$

where the functions $i_{n}:=i_{n}^{1}$ and $j_{n}:=j_{n}^{1}$ are given by

$$
\begin{aligned}
& i_{n}(x)=\left|1+x \frac{h_{n}^{\prime}}{h_{n}}(x)\right|^{2} h_{n}(x), \\
& j_{n}(x)=i_{n}(x)+\left|2+4 x \frac{h_{n}^{\prime}}{h_{n}}(x)+x^{2} \frac{h_{n}^{\prime \prime}}{h_{n}}(x)\right| h_{n}(x) .
\end{aligned}
$$

Making a change of variables, we see that the quantities $I_{n}^{\theta}$ and $\widetilde{I}_{n}(\chi)$, defined in (27), satisfy

$$
\begin{aligned}
I_{n}^{\theta} & =\frac{1}{\theta^{2}} \int_{K} i_{n}(x) \mathrm{d} x=\frac{I_{n}}{\theta^{2}}, \\
\widetilde{I}_{n}(\chi) & =\sup _{|\zeta| \leq \chi} \frac{I_{n}}{\left([\zeta]_{n}\right)^{2}} \leq \frac{I_{n}}{\left(\left|\theta_{0}\right|-(\chi / \sqrt{n})\right)^{2}} .
\end{aligned}
$$

Using Cauchy-Schwarz inequality and again a change of variables, we obtain the following: for all $\chi>0$, the quantities $\widetilde{J}_{n}^{\rho}(\chi), \rho \in(0,1)$, defined in $(28)$, satisfy

$$
\begin{aligned}
\widetilde{J}_{n}^{\rho}(\chi) & \leq \sup _{|\zeta|,|\xi| \leq \chi}\left[\int_{K} \frac{j_{n}^{[\zeta]_{n}}(x)^{2}}{h_{n}^{[\zeta]_{n}}(x)^{2 \rho}} \mathrm{d} x\right]^{1 / 2}\left[\int_{K} \frac{j_{n}^{[\varepsilon]_{n}}(x)^{2}}{h_{n}^{[\varepsilon]_{n}}(x)^{2 \rho^{\prime}}} \mathrm{d} x\right]^{1 / 2} \\
& =\sup _{|\zeta|,|\varepsilon| \leq \chi}\left[\frac{1}{\left|[\zeta]_{n}\right|^{5-2 \rho}\left|[\varepsilon]_{n}\right|^{5-2 \rho}} \int_{K} \frac{j_{n}(x)^{2}}{h_{n}(x)^{2 \rho}} \mathrm{d} x \int_{K} \frac{j_{n}(x)^{2}}{h_{n}(x)^{2 \rho^{\prime}}} \mathrm{d} x\right]^{1 / 2} \\
& \leq|| \theta_{0}\left|-\frac{\chi}{\sqrt{n}}\right|^{4 \rho-10}\left[J_{n}(\rho) J_{n}\left(\rho^{\prime}\right)\right]^{1 / 2}, \quad J_{n}(r):=\int_{K} \frac{j_{n}(x)^{2}}{h_{n}(x)^{2 r}} \mathrm{~d} x .
\end{aligned}
$$


Corollary 1. For the scale model (5), the sequence of statistical models $E^{n}$ has the LAN property with speed $\sqrt{n}$ at each $\theta \in \Theta$ if the following conditions are satisfied:

(C0): under $\mathbb{P}_{1}$, the support $K$ of the law of $X_{1}$ is either $\mathbb{R}$ or $\mathbb{R}_{+}$or $\mathbb{R}_{-}$. For all

$t>0$, the r.v. $X_{t}$ has a p.d.f. $g_{t}^{1}(x)$ which is of class $C^{2}$ on the interior of $K$.

(C1): the sequence $I_{n}:=\int_{K} i_{n}(x) \mathrm{d} x$ satisfies $\lim _{n \longrightarrow+\infty} I_{n}=I \in(0, \infty)$,

(C2): there exists $a \in\left(0, \frac{1}{2}\right)$ such that $\lim _{n \longrightarrow \infty} \frac{1}{n} J_{n}(\rho)=0 \quad$ for $\rho \in\left\{\frac{1}{2}, a, 1-a\right\}$.

In this case, the asymptotic Fisher information quantity is $I(\theta)=I / \theta^{2}$.

Proof. It is immediate that (C0) implies (H0). Representation (63) shows that (C1) implies (H1) and (64) shows that (C2) implies (H2).

Remark 2. Since $h_{n}$ is the p.d.f. of $X_{u_{n}}$, then we should be aware that the discretization path $u_{n}$ intervene in the assumptions (C1) and (C2). In Theorem 2, we will see that in some favorable cases, the effect of the path $u_{n}$ has a quite negligible effect. In general, the LAN property depends strongly on the asymptotic of $u_{n}$.

\section{Examples of LAN Property for Lévy Processes Attracted by Stable Processes}

In this section, we provide some examples of Lévy processes $X$ satisfying the conditions of Corollary 1 . This corollary provides the LAN property for the scale model (5), $X=\theta Y$, under regularity and integrability conditions on the p.d.f. of $Y_{t}, t>0$. Unfortunately, these p.d.f., if they exist, are not explicit in general; for this reason, we focus on processes $Y$ which belong to the domain of attraction of stable processes.

We recall that a process $S^{\alpha, \beta, \gamma, \delta}$ is stable, if it is a Lévy process with Lévy exponent given in (10). In the case $\alpha \in(0,1)$ and $\delta \geq 0$, the process $S^{\alpha, 1, \gamma, \delta}$ is a subordinator, i.e., a positive increasing the Lévy process, and the distribution of $S_{t}^{\alpha, 1, \gamma, \delta}, t>0$, has a support equal to $[\delta t, \infty)$. In all cases, $S_{t}^{\alpha, 1, \gamma, \delta}, t>0$, has a p.d.f. $G_{t}^{\alpha, \beta, \gamma, \delta}$ which is infinitely differentiable and is explicit only for the particular values $(\alpha, \beta)=((1 / 2), 1),(1,0),(2,0)$. These values correspond, respectively, to the first passage times of the Brownian motion, the Cauchy process, and the Brownian Motion. Otherwise, $G_{t}^{\alpha, \beta, \gamma, \delta}$ is be expressed only as the inverse Fourier transform of $\exp \phi(u)$. As announced, the introduction, we focus in this section on the case where the Lévy processes $Y$, in the scale model (5), is attracted by a stable process, i.e., satisfying (13). Bertoin and Doney [22] showed that if (10) holds, then the process $Y$ is attracted by a stable one, and then, the following holds:

(i) There exists $b \in \mathbb{R}, \alpha \in(0,2]$, and a slowly varying function $l(t)$ (i.e., a function satisfying $(l(\lambda t) / l(t)) \longrightarrow 1, \forall \lambda>0)$, such that $b(t)=b t$ and $a(t)=t^{1 / \alpha} l(t)$

(ii) $v(\mathrm{~d} x)=G(x) \mathrm{d} x$, where $G$ is the p.d.f. of some stable r.v. $S_{1}^{\alpha, \beta, \gamma, 0}, \beta \in[-1,1], \gamma>0$;

(iii) The p.d.f. $G_{t}$ of $\widehat{Y}_{t}, t>0$, is infinitely differentiable and satisfies

$$
\left(G_{t}\right)^{(k)}(x) \longrightarrow G^{(k)}(x), \quad \text { uniformly in } x \in \operatorname{Support}(G), t \longrightarrow 0 \text { or } t \longrightarrow \infty, k \in \mathbb{N}
$$

(iv) The convergence in (13) can be entirely expressed with the behavior of the tail of the Lévy measure of $Y$ or by the existence of a Brownian component.

Observe that the p.d.f. $h_{n}$ of $Y_{u_{n}}$ is represented by

$$
h(x)=G_{u_{n}}\left(\frac{x-b\left(u_{n}\right)}{a\left(u_{n}\right), \quad x \in \mathbb{R} .}\right.
$$

As in (8), let us denote by $\bar{G}_{u_{n}}$ and $\bar{G}$ the logarithmic derivative of $G_{u_{n}}$ and $\log G$, respectively. After the change of variable $x \mapsto a\left(u_{n}\right) x+b\left(u_{n}\right)$, the asymptotic Fisher information quantity in (63) takes the form

$$
I(\theta)=\frac{I}{\theta^{2}}, \quad I=\lim _{n \longrightarrow \infty} \int\left[1+\left(x+\frac{b\left(u_{n}\right)}{a\left(u_{n}\right)}\right) \bar{G}_{u_{n}}(x)\right]^{2} G_{u_{n}}(x) \mathrm{d} x .
$$

The convergence (66) does not ensure that $I(\theta)$ is finite and nonnull; it only ensures that if it happens, then 


$$
\begin{aligned}
& l=\lim _{n \longrightarrow \infty}\left(\frac{b\left(u_{n}\right)}{a\left(u_{n}\right)}\right) \text { exists, } \\
& I=\int[1+(x+l) \bar{G}(x)]^{2} G(x) \mathrm{d} x \in(0, \infty) .
\end{aligned}
$$

The stronger control

$$
\limsup _{|x| \longrightarrow \infty, n \longrightarrow \infty}\left|x \bar{G}_{u_{n}}(x)\right|<\infty,
$$

is sufficient to prove (69), but it is hard to obtain. In [14, 17], we provided controls for the p.d.f. $G_{t}(x)$ in the space variable $x$ and uniformly in small or big time $t$, the processes

$$
\begin{aligned}
Y & =\sum_{i=k}^{N} S^{a_{k}, b_{k}, c_{k}, d_{k}}, \\
\text { or } Y & =S^{\alpha, \beta, 1,0} \circ Z,
\end{aligned}
$$

$$
\frac{Y_{t}-\sum_{k=1}^{N} \delta_{t, a_{k}}}{\left(\gamma_{i} t\right)^{1 / a_{i}}} \stackrel{d}{\longrightarrow} S_{1}^{a_{i}, b_{i}, 1,0}
$$

The last property will be useful in Subsection 4.2 when taking the path $u_{n} \longrightarrow 0$ (respectively, $\left.u_{n} \longrightarrow \infty\right)$.

(ii) If $Y=S^{\alpha, \beta, 1,0} \circ Z$, we assume that the subordinator $Z$ is itself attracted by a stable subordinator: for some $0<\epsilon<1$ and some (deterministic) speed $r_{t}$,

$$
\left.\frac{Z_{t}}{r_{t}} \stackrel{d}{\longrightarrow} S_{1}^{\mathcal{\varepsilon}, 1,1,0}, \quad \text { as } t \longrightarrow 0 \text { (respectively, as } \infty\right) \text {. }
$$

The function $t \mapsto r_{t}$ is necessarily a regularly varying function of order $1 / \epsilon$ at 0 (respectively, $\infty$ ): $r_{t}=t^{1 / \epsilon} l(t)$ and $l$ is slowly varying, i.e., $(l(\lambda t) / l(t)) \longrightarrow 1, \forall \lambda>0$. Then, there exist $\beta \in(-1,1)$ and $\gamma^{\prime}>0$, such that

$$
\frac{Y_{t}}{r_{t}^{1 / \alpha}} \stackrel{d}{\longrightarrow} S_{1}^{\varepsilon \alpha, \beta^{\prime}, \gamma^{\prime}, 0}, \quad \text { as } t \longrightarrow 0(\text { respectively, } t \longrightarrow \infty) \text {. }
$$

4.1. The Scale Models Associated with Stable Processes Have the LAN Property. The following result is a first application of Corollary 1.

Theorem 2. For the scale model (5), assume $Y=S^{\alpha, \beta, \gamma, \delta}$ is a stable process, such that $\delta=0$ is null if $\alpha<1$ and $|\beta|=1$. Recall $\delta_{t \alpha}$ is defined in (12). Let the path $u_{n}$ satisfy $l_{n}=$ $\left(\gamma u_{n}\right)^{-1 / \alpha} \delta_{u_{n}, \alpha} \longrightarrow l \in \mathbb{R}$ and one of the following conditions:

(i) $u_{n} \longrightarrow L \in(0, \infty)$

(ii) $u_{n} \longrightarrow L=0$ and $n^{R} u_{n} \longrightarrow \infty$, for some $R>0$

(iii) $u_{n} \longrightarrow L=+\infty$ and $n^{-S} u_{n} \longrightarrow 0$, for some $S>0$ where the coefficients $\left(a_{k}, b_{k}, c_{k}, d_{k}\right)$ vary in a set $\mathscr{D}_{N}$, the processes $S^{a_{k}, b_{k}, c_{k}, d_{k}}$ are independent, and $S^{\alpha, \beta, 1}$ is independent of the subordinator $Z$. We emphasize that in (71), $Y$ satisfies (13) and does not enjoy the scaling property (12) anymore.

(i) If $Y=\sum_{i=k}^{N} S^{a_{k}, b_{k}, c_{k}, d_{k}}$ and the stability coefficients $a_{k}$ are pairwise different, we have an asymptotic scaling property; if $i_{\wedge}=\operatorname{Arg} \min \left\{\alpha_{i}, 1 \leq i \leq N\right\} \quad$ and $i_{\vee}=\operatorname{Arg} \max \left\{\alpha_{i}, 1 \leq i \leq N\right\}$ and $i=i_{\vee}$ (respectively, $\left.i_{\wedge}\right)$, then

as $t \longrightarrow 0$ (respectively, $t \longrightarrow \infty)$.

Then, the sequence of sequence of filtered statistical scale models $E^{n}(15)$ have the LAN property with speed $\sqrt{n}$ at each $\theta \in \Theta$. The asymptotic Fisher information quantity is given by

$$
I(\theta)=\frac{1}{\theta^{2}}\left[\int(y+l)^{2} \frac{G^{\prime}(y)^{2}}{G(y)} \mathrm{d} y-1\right]
$$

where $G$ is the p.d.f. of $S_{1}^{\alpha, \beta, 1,0}$.

Remark 3. If $\alpha \in(0,1)$, then support of the law of $S_{1}^{\alpha, \beta, \gamma, \delta}$ is $[\delta, \infty)$ (respectively, $(-\infty, \delta]$ ) if $\beta=1$ (respectively, $\beta=-1$ ); otherwise, the support is whole $\mathbb{R}$. The assumption $\delta=0$ ensures that the support of the law of $S_{1}^{\alpha, \beta, \gamma, \delta}$ satisfies the condition $(\mathrm{C} 0)$ of Corollary 1 . We are aware that the cases $n u_{n} \longrightarrow 0$ and $u_{n} \longrightarrow \infty$ are not very realistic by the statistical point of view. The most interesting cases are as follows:

(a) $u_{n}=u \in(0, \infty)$, then $L=u$, and the assumptions of Theorem 2 are satisfied. This is an essentially trivial result because we treat then a regular i.i.d. model.

(b) $u_{n}=(u / n), u \in(0, \infty)$, then $L=0$, and the assumptions of Theorem 2 are satisfied if and only if one of the following holds:

(i) $\alpha<1, \delta=0$, and then, $l=0$;

(ii) $\alpha=1, \beta=0$, and then, $l=\delta / \gamma$;

(iii) $\alpha>1$, and then, $l=0$.

Proof. of Theorem 2. (1a) Recall that $h_{n}$ denotes the infinitely differentiable p.d.f of $S_{u_{n}}^{\alpha, \beta, \gamma, \delta}$. Due to the scaling property (12), we have the following representations: for all $k \in \mathbb{N}$ and $x \in \operatorname{support}\left(h_{n}\right)$, 


$$
\left(h_{n}\right)^{(k)}(x)=\frac{1}{\left(\gamma u_{n}\right)^{(k+1) / \alpha}}\left(G_{1}^{\alpha, \beta, 1,0}\right)^{(k)}\left(\frac{x-\delta_{u_{n}, \alpha}}{\left(\gamma u_{n}\right)^{1 / \alpha}}\right),
$$

where

$$
\operatorname{Support}\left(h_{n}\right)= \begin{cases}\mathbb{R}_{+}, & \text {if } 0<\alpha<1, \beta=1, \\ \mathbb{R}_{-}, & \text {if } 0<\alpha<1, \beta=-1, \\ \mathbb{R}, & \text { otherwise. }\end{cases}
$$

(1b) In $[14,17]$, we provided several properties of the p.d.f. $G=G_{1}^{\alpha, \beta, 1,0}$. For instance, there exist positive constants $A, B, C, D$ that depend explicitly on $\alpha, \beta$, such that

$$
\begin{aligned}
& \tilde{0+}(\text { respectively, }\underset{0-}{\sim}) \xi(x), \quad \text { if } \beta=1(\text { respectively, }-1) \text { and } 0<\alpha<1, \\
& G(x)\underset{+\infty}{\sim}(\text { respectively, } \underset{-\infty}{\sim}) \chi(x), \quad \text { if } \beta \neq-1 \text { (respectively, } 1) \text { and } 0<\alpha<2, \\
&\underset{+\infty}{\sim}(\text { respectively, } \underset{-\infty}{\sim}) \eta(x), \quad \text { if } \beta=-1 \text { (respectively, } 1) \text { and } \alpha=1, \\
&\underset{+\infty}{\sim}(\text { respectively, } \underset{-\infty}{\sim}) \xi(x), \quad \text { if } \beta=-1 \text { (respectively, } 1) \text { and } 1<\alpha \leq 2 .
\end{aligned}
$$

where

$$
\begin{aligned}
& \chi(x):=\frac{D}{|x|^{\alpha+1}} \\
& \xi(x):=B|x|^{(2-\alpha) / 2(\alpha-1)} e^{-A|x|^{\alpha / \alpha-1)}}, \\
& \eta(x)=C \exp \left(-e^{\pi|x| / 2}+\frac{\pi|x|}{4}\right),
\end{aligned}
$$

$$
\begin{aligned}
& \left.\widetilde{0+}\left(\text { respectively, } \underset{0_{-}}{\sim}\right) a|x|^{k /(\alpha-1)}, \quad \text { if } \beta=1 \text { (respectively, }-1\right) \text { and } 0<\alpha<1 \text {, } \\
& \underset{+\infty}{\sim}(\text { respectively, } \underset{-\infty}{\sim}) b|x|^{-k}, \quad \text { if } \beta \neq-1 \text { (respectively, } 1 \text { )and } 0<\alpha<2,
\end{aligned}
$$$$
F_{k}(x)
$$$$
\underset{+\infty}{\sim}(\text { respectively, } \underset{-\infty}{\sim}) c \exp \left(\frac{k \pi|x|}{2}\right), \quad \text { if } \beta=-1(\text { respectively, } 1) \text { and } \alpha=1,
$$$$
\left.\underset{+\infty}{\sim}(\text { respectively, } \underset{-\infty}{\sim}) a|x|^{k /(\alpha-1)}, \quad \text { if } \beta=-1 \text { (respectively, } 1\right) \text { and } 1<\alpha \leq 2 \text {. }
$$

are continuous on support $\left(h_{n}\right)$, given by (77), and there exist positive numbers $a, b, c$, depending explicitly on $\alpha$ and $\beta$ and $k$, such that
From the last equivalences, we see that for any nonnegative integer $s$, we have the implication

$$
0 \leq r \leq k \Longrightarrow \lim _{|x| \longrightarrow \infty}|x|^{2(1-\rho)(1+\alpha)}\left(|x|^{r} F_{k}\right)^{s}(G)^{2(1-\rho)} \in[0, \infty) .
$$

Thus, since $0<\rho<1-(1 /(2(1+\alpha))) \Leftrightarrow 2(1-\rho)(1+$ $\alpha)>1$, then
$0 \leq r \leq k$

$0<\rho<1-\frac{1}{(\alpha+1)} \Longrightarrow x \mapsto\left(|x|^{r} F_{k}(x)\right)^{s} G(x)^{2(1-\rho)} \in L^{1}(\mathrm{~d} x)$.

(2) We need to verify the assumptions of Corollary 1, i.e., to check $(\mathbf{C 1})$ : 


$$
\begin{aligned}
I_{n} & =\int\left[1+x \frac{h_{n}^{\prime}}{h_{n}}(x)\right]^{2} h_{n}(x) \mathrm{d} x \longrightarrow I \\
& =\int(y+l)^{2} \frac{\left(G^{\prime}\right)^{2}}{G}(y) \mathrm{d} y-1 \in(0, \infty), \quad \text { as } n \longrightarrow \infty,
\end{aligned}
$$

and also (C2); there exists $a \in(0,(1 / 2))$, such that for $\rho \in\{(1 / 2), a, 1-a\}$, one has

$$
\begin{aligned}
\frac{1}{n} J_{n}(\rho) & =\frac{1}{n} \int \frac{\left(j_{n}\right)^{2}}{\left(h_{n}\right)^{2 \rho}}(x) \mathrm{d} x \longrightarrow 0, \quad j_{n}(x) \\
& =\left[\left|1+x \frac{h_{n}^{\prime}}{h_{n}}(x)\right|^{2}+\left|2+4 x \frac{h_{n}^{\prime}}{h_{n}}(x)+x^{2} \frac{h_{n}^{\prime \prime}}{h_{n}}(x)\right|\right] h_{n}(x) .
\end{aligned}
$$

(3) The scaling property (76) and the corresponding change of variables give the following representation of the quantity $I_{n}$ in (84):

$$
I_{n}=\int\left[1+\left(y+l_{n}\right) \frac{G^{\prime}(y)}{G(y)}\right]^{2} G(y) \mathrm{d} y .
$$

Using (82) and the fact that $l_{n} \longrightarrow l \in \mathbb{R}$, we obtain

$$
\begin{gathered}
x \mapsto \sup _{n \in \mathbb{N}}\left[1+\left(y+l_{n}\right) \frac{G^{\prime}}{G}(y)\right]^{2} G(y) \in L^{1}(\mathrm{~d} y), \\
\lim _{n \longrightarrow \infty} I_{n}=\int\left[1+(y+l) \frac{G^{\prime}}{G}(y)\right]^{2} G(y) \mathrm{d} y .
\end{gathered}
$$

Developing the last expression, integrating by parts and using the fact that $G(y)$ and $y G(y)$ both tend to 0 as $y$ goes to each endpoint of the support (77), we recover (84).

(4) Again, by the change of variables corresponding to (76) and by the representation (85), one has

$$
J_{n}(\rho)=\left(\gamma u_{n}\right)^{(2 \rho-1) / \alpha} \int\left[\left|1+\left(y+l_{n}\right) \frac{G^{\prime}}{G}(y)\right|^{2}+\left|2+4\left(y+l_{n}\right) \frac{G^{\prime}}{G}(y)+\left(y+l_{n}\right)^{2} \frac{G^{\prime \prime}}{G}(y)\right|\right]^{2} G(y)^{2(1-\rho)} \mathrm{d} y
$$

Thanks to (83) and to the fact that $l_{n} \longrightarrow l \in \mathbb{R}$, and it is clear that if $\rho \in(0,1-1 / 2(\alpha+1))$, then

$$
\sup _{n \in \mathbb{N}}\left[\left|1+\left(y+l_{n}\right) \frac{G^{\prime}}{G}(y)\right|^{2}+\mid 2+4\left(y+l_{n}\right) \frac{G^{\prime}}{G}(y)+\left(y+l_{n}^{2} \frac{G^{\prime \prime}}{G}(y) \mid\right]^{2} G(y)^{2(1-\rho)}\right.
$$

is integrable. Let $\epsilon:=(1 / 2)-(1 /(2(\alpha+1)))$. To prove the convergence (85), it is enough to have

$$
\begin{gathered}
\rho \in\left(0, \epsilon+\frac{1}{2}\right), \\
\lim _{x \rightarrow \infty} \frac{u_{n}^{(2 \rho-1) / \alpha}}{n}=0 .
\end{gathered}
$$

(5) Now, we distinguish between the values of $L=\lim _{n \longrightarrow \infty} u_{n}$.

(a) If $L=0$ and if $\rho \geq(1 / 2)$, then (91) is true. By assumptions, we have $\left(1 / u_{n}\right) \leq n^{R}$, for large $n$. If $\rho<(1 / 2)$, then we have (91) as soon as $(R(1-2 \rho) / \alpha)<1$, which is equivalent to $\rho>(1 / 2)-(\alpha /(2 R))$. Thus, we only have to choose $\epsilon^{\prime}=(\epsilon \wedge \alpha /(4 R))$ and $a=(1 / 2)-\epsilon^{\prime}$ to get (90) to obtain (91) for $\rho \in\{(1 / 2), a, 1-a\}$.

(b) If $L \in(0, \infty)$, then (91) is always true.

(c) If $L=+\infty$ and if $\rho \leq(1 / 2)$, then (91) is true. As in (5a), we have $u_{n} \leq n^{S}$ for large $n$. If $\rho>(1 / 2)$ then we have (91) as soon as $(S(2 \rho-1) / \alpha)<1$, which is equivalent to $\rho<(1 / 2)+(\alpha /(2 S))$. Thus, we only need to choose $\epsilon^{\prime \prime}=(\epsilon \wedge \alpha /(4 S))$ and $a=(1 / 2)-\epsilon^{\prime \prime}$ to obtain (90) and (91) for $\rho \in\{1 / 2, a, 1-a\}$.

4.2. The Scale Models Associated with the Sum of Independent Stables Processes Have the LAN Property. This subsection gives a second example which also generalizes the previous one and achieves the situation (13). Define $\bar{K}(\alpha)=1$ if $\alpha \leq 1$ 
and $\bar{K}(\alpha)=(\alpha-2) / \alpha$ and assume the following restrictions on the skewness parameters:

$\left(S_{\mathrm{a}, \mathrm{b}}\right)$ : we have $N$ independent stable processes $S^{a_{k}, b_{k}, c_{k}, 0}$, such that the parameters satisfy

$$
\begin{aligned}
& \text { (a) } a_{1}<a_{2}<\cdots<a_{N}<2 \text { and } \mathscr{D}=\stackrel{N}{N=1}_{n}\left[a_{k}, \frac{2}{\left(1+\left|b_{k} \bar{K}\left(a_{k}\right)\right|\right)}\right] \neq \varnothing ; \\
& \text { (b) } b_{k}=0 \text { if } a_{k}=1 \text { and } B=\max \left\{\left|\frac{b_{k} \bar{K}\left(a_{k}\right)}{\bar{K}\left(a_{N}\right)}\right|, \quad 1 \leq k \leq N\right\}<1 .
\end{aligned}
$$

Let $Y=\sum_{k=1}^{N} S_{k}^{a_{k}, b_{k}, c_{k}, 0}$ and $Y^{i}$ be the processes defined by

$$
Y_{t}^{i}=\frac{Y_{t}}{t^{1 / a_{i}}}, \quad i=1 \text { or } i=N \text { and }, t>0 \text {. }
$$

The processes $Y^{i}$ satisfy the asymptotic scaling property (72). Denote by $H_{t}$ the p.d.f of $Y_{t}$,

$$
H_{t}=G_{t}^{a_{1}, b_{1}, c_{1}, 0} * \cdots * G_{t}^{a_{N}, b_{N}, c_{N}, 0},
$$

and by $H_{i, t}$, the one of $Y_{t}^{i}$. Then, $h_{n}=H_{u_{n}}$ satisfies

$$
\left(h_{n}\right)^{(k)}(x)=\left(u_{n} c_{i}\right)^{-(k+1) / \alpha_{i}}\left(H_{i, u_{n}}\right)^{(k)}\left(\left(u_{n} c_{i}\right)^{-1 / a_{i}} x\right) .
$$

In $[14,17]$, we showed that the following functions $H_{0}$ and $H_{\infty}$ given by

$$
H_{L}:= \begin{cases}\lim _{t \longrightarrow \infty} H_{1, t}(x)=G_{1}^{a_{1}, b_{1}, 1,0}(x), & \text { if } L=+\infty, \\ \lim _{t \longrightarrow 0+} H_{N, t}(x)=G_{1}^{a_{N}, b_{N}, 1,0}(x), & \text { if } L=0,\end{cases}
$$

are well defined, and the above convergence holds uniformly in $x \in \operatorname{support}\left(G_{1}^{a_{i}, b_{i}, 1,0}\right)$ and still hold for the successive derivatives. As one can guess, we are going to exploit identity (95) and state the following result.

Theorem 3. Let $\left(u_{n}\right)_{n}$ be a sequence satisfying one of the following conditions:

(i) $u_{n} \longrightarrow L \in(0, \infty)$ (ii) $u_{n} \longrightarrow L=0$, and there exists $R>0$, such that
$n^{R} u_{n} \longrightarrow+\infty$;

(iii) $u_{n} \longrightarrow L=+\infty$, and there exists $S>0$, such that $n^{-S} u_{n} \longrightarrow 0$.

For the scale model (5) with $Y=\sum_{k=1}^{N} S_{k} a_{k}, b_{k}, c_{k}, 0$, assume $\left(S_{a, b}\right)$. Then, the sequence of filtered statistical scale models $E^{n}(15)$ have the LAN property with speed $\sqrt{n}$ at each value $\theta \in \Theta$. With $H_{L}$ given in (95), the asymptotic Fisher information quantity has the following expression:

$$
\begin{aligned}
I\left(\theta_{0}\right) & =\frac{I_{L}}{\theta^{2}}, \\
I_{L} & =\int y^{2} \frac{\left(H_{L}^{\prime}\right)^{2}(y)}{H_{L}(y)} \mathrm{d} y-1 .
\end{aligned}
$$

\section{Remark 4}

(i) Let us briefly explain the nature of the assumption in Theorem 3. In [17], conditions of type $\left(S_{a, b}\right)$ allowed us to show that $Y_{t}^{i}$ is distributed as an $\alpha$-stable variable mixed on the skewness and scale parameters by other processes. More precisely, for all $t>0$, we have these identities in distribution: for all $\alpha$ in the interior of the domain $\mathscr{D}$ (given in $\left(S_{a, b}\right)$ ) and for all $t>0$, there exist a r.v. $\beta_{t}$ and $\gamma_{t}^{i}$, such that

$$
Y_{t}^{i} \stackrel{d}{=} S_{1}^{\alpha, \beta_{t}, \gamma_{t}^{i}, 0} \stackrel{d}{=}\left(\gamma_{t}^{i}\right)^{1 / \alpha} S_{1}^{\alpha, \beta_{t}, 1,0} .
$$

The processes $\beta$ and $\gamma^{i}$ are

$$
\begin{gathered}
\left|\beta_{t}\right| \leq B, \\
\frac{C}{t^{\alpha / a_{i}}} Z_{t} \leq \gamma_{t}^{i} \leq \frac{D}{t^{\alpha / a_{i}}} Z_{t},
\end{gathered}
$$

Where $Z_{t}=\sum_{k=1}^{n} S_{t}^{\left(a_{k} / \alpha\right), 1,1,0}$ is a sum of independent standard stable subordinators, and the nonnegative numbers $C<D$ depend only on $\left(\alpha, a_{1}, b_{1}, c_{1}, \ldots, a_{N}, b_{N}, c_{N}\right)$. In the case where $b_{k} \bar{K}\left(a_{k}\right)$ is a constant for all $k=1, \ldots, N$, then $\beta_{t}=b_{1} \bar{K}\left(a_{1}\right), \gamma_{t}^{i}$ is distributed as a normalized sum of independent stable subordinators, and the converge in the following distribution holds.

$$
\begin{aligned}
& \left(\beta_{t}, \gamma_{t}^{i}\right) \longrightarrow\left(\frac{b_{i} \bar{K}\left(a_{i}\right)}{\bar{K}(\alpha)}, S_{1}^{\left(a_{i} / \alpha\right), 1,1,0}\right), \\
& \quad \text { if } i=1 \text { and } t \longrightarrow \infty \text {, or if } i=N \text { and } t \longrightarrow 0 .
\end{aligned}
$$


Furthermore, notice that the assumption $\left(\mathbf{S}_{\mathbf{a}, \mathbf{b}}\right)$ is satisfied in the symmetrical cases

(ii) In general, when $\beta, \gamma, \delta$ are r.v.'s lying in the set of admissible parameters and $\mathscr{F}$ is the $\sigma$-field generated by them, we gave in [17] a structure of the $\mathscr{F}$-conditional Lévy process to $\left(S_{r}^{\alpha, \beta, \gamma, \delta}\right)_{r \geq 0}$. See also [13] for the notion of the $\mathscr{F}$-conditional Lévy process. If $\beta$ is deterministic, $S_{1}^{\alpha, \beta, \gamma, 0}$ is simply distributed as $\gamma^{1 / \alpha} S_{1}^{\alpha, \beta, 1,0}$, and with our construction, we allow the same identity even if $\beta$ is random and correlated with $\gamma$. We also considered in [17] the densities of some families of mixed stable variables $\left(S_{1}^{\alpha, \beta_{t}, \gamma_{t}, 0}\right)_{t \in T}$ and gave several examples when these densities and their derivatives behave like the proper stable densities and this uniformly in $t \in T$.

(iii) It is also possible to state a version of Theorem 3 with stable processes with drifts. It is enough to strengthen the conditions on the asymptotic as done in Theorem 2. We consider that Theorem 3 is far from being exhaustive. It is produced in the aim of illustrating the difficulty of this case. If one wants to reduce the assumption $\left(\mathbf{S}_{\mathbf{a}, \mathbf{b}}\right)$ on the coefficients, then additional controls on the densities are needed.

Before tackling the proof of Theorem 3, we need the following result borrowed from [17].

Theorem 4 (See [17]). Controls of the densities of some mixed stable variables $\left(S_{1}^{\alpha, \beta_{r}, \gamma_{r}, 0}\right)_{r \in \mathscr{R}}$. Let $\left(\gamma_{r}\right)_{r \geq 0}$ be a pure jump subordinator characterized by

$$
\mathbb{E}\left[e^{-\lambda \gamma_{1}}\right]=\exp \int_{(0, \infty)}\left(e^{-\lambda x}-1\right) \nu(\mathrm{d} x), \quad \lambda \geq 0 .
$$

Assume $v(x)=v(x, \infty)=x^{-a} L(x)$, where $a \in(0,1)$, and $L$ is a slowly varying function. For $r, x>0$, define

$$
\begin{aligned}
v_{r} & :=\sup \left\{t>0: v(t)>\frac{1}{r}\right\}, \\
v_{r}(x) & :=r \nu\left(x v_{r}, \infty\right), \\
\bar{\gamma}_{r} & :=\frac{\gamma_{r}}{v_{r}} .
\end{aligned}
$$

(a) If $L$ is slowly varying at infinity and $r \longrightarrow \infty$ or if $L$ is slowly varying at zero, then

$$
\begin{aligned}
& \nu_{r}(x) \longrightarrow \frac{1}{x^{a}}, \quad x>0, \\
& \bar{\gamma}_{r} \stackrel{d}{\longrightarrow} S_{1}^{a, 1,(\Gamma(1-a) / a), 0}, \quad \text { as } r \longrightarrow 0+.
\end{aligned}
$$

(b) Moreover, assume that there exist $0<c \leq a \leq d<1$ and $K \geq 1$, such that

$$
\left(\frac{y}{x}\right)^{a-c} \leq \frac{L(y)}{L(x)} \leq K\left(\frac{y}{x}\right)^{a-d}, \quad 0<y<x .
$$

Let $R^{\prime}>R>0$ and $\left(\gamma_{r}^{p}\right)_{t \in R_{p}}$ denote one of these families:

$R_{1}=R_{4}=(0, R], R_{2}=\left[R, R^{\prime}\right], R_{3}=[R, \infty)$, and $\gamma_{r}^{1}=\gamma_{r}^{2}=\gamma_{r}, \gamma_{r}^{3}=\bar{\gamma}_{r}$ with $L$ slowly varying at $\infty$, and $\gamma_{r}^{4}=\bar{\gamma}_{r}$, with $L$ slowly varying at 0 . Let $\alpha \in(0,2)$ and $\left(\beta_{r}\right)_{r \geq 0}$ be any family of r.v.'s, such that $\sup _{r \geq 0}\left|\beta_{r}\right| \leq B$, for some $B \in[0,1), \quad(B=0$, if $\alpha=1)$. Then, the p.d.f. $G_{r}^{p}$ of the mixed stable variables $S_{1}^{\alpha, \beta_{r}, \gamma_{r}}$ are infinitely differentiable and satisfy the following: for all $k \in \mathbb{N}$ and all $p$, we have

$$
\begin{aligned}
& 0<\liminf _{|x| \longrightarrow \infty} \inf _{r \in R_{p}}|x|^{1+\alpha d} G_{r}^{p}(x), \\
& \limsup _{|x| \longrightarrow \infty} \sup _{r \in R_{p}}|x|^{1+\alpha c} G_{r}^{p}(x)<\infty, \\
& \limsup _{|x| \longrightarrow \infty} \sup _{r \in R_{p}, x \in \mathbb{R}} \frac{\left|x^{k}\left(G_{r}^{p}\right)^{(k)}(x)\right|}{G_{r}^{p}(x)}<\infty .
\end{aligned}
$$

For all $X>0, p \neq 1$, we have

(c) If $\left(\gamma_{r}^{\prime}\right)_{r \geq 0}$ is a family of r.v.'s, such that $\left(\gamma_{r} / K\right) \leq \gamma_{r}^{\prime} \leq K \gamma_{r}$ for some $K>1$ and all $r \geq 0$, then the controls (106) and (17) remain true for the p.d.f.'s obtained by replacing $\left(\gamma_{r}, \bar{\gamma}_{r}\right)$ by $\left(\gamma_{r}^{\prime},\left(\gamma_{r}^{\prime} / v_{r}\right)\right)$, where $v_{r}$ is given by (103).

Remark 5. If $\gamma$ is a pure jump a-stable subordinator, then $\nu_{r}(x)=x^{-a}$, and the scaling property gives $\bar{\gamma}_{r} \stackrel{d}{=} S_{1}^{a, 1, \Gamma(1-a) / a}$. Furthermore, if $\beta_{r}=\beta, r>0$, is deterministic, then $S_{1}^{\alpha, \beta_{r}, \bar{\gamma}_{r}} \stackrel{d}{=} S_{1}^{\alpha a, \beta^{\prime}, \gamma^{\prime}}$, for some $\beta^{\prime} \in(-1,1), \gamma^{\prime}>0$. The estimates (106) and (17) are an immediate consequence of the behavior of the stable densities given in the Proof of Theorem 2.

Example 1. The following are examples of processes satisfying the conditions of Theorem 4 . Let $0<b, b_{1}, \ldots, b_{N}<1$, and $c, c_{1}, \ldots, c_{N}>0$. Let $\gamma^{1}, \gamma^{2}$ be pure jump subordinators whose Lévy measures are, respectively, equal to

$$
\begin{aligned}
& \nu_{1}(\mathrm{~d} x)=\sum_{k=1}^{N} \frac{c_{i}}{x^{b_{k}+1}} 1 l_{x>0} \mathrm{~d} x, \\
& \nu_{2}(\mathrm{~d} x)=\frac{c}{x^{b+1}} 1 l_{x>0} \mathrm{~d} x+\delta_{1}(\mathrm{~d} x) .
\end{aligned}
$$

The process $\gamma^{1}$ is the sum of independent stable subordinators, and $\gamma^{2}$ is the independent sum of a stable subordinator and a standard Poisson process. With $b_{\vee}=$ $\max b_{i}$ and $b_{\wedge}=\min b_{i}$, notice that

$$
\begin{aligned}
& \nu_{1}(x)=\frac{L_{1}^{\vee}(x)}{x^{b_{\vee}}}=\frac{L_{1}^{\wedge}(x)}{x^{b_{\wedge}}}, \\
& \nu_{2}(x)=\frac{L_{2}(x)}{x^{b}},
\end{aligned}
$$

where $L_{1}^{\vee}, L_{2}$ are slowly varying at $0, L_{1}$ is slowly varying at $\infty$, and 


$$
\begin{aligned}
0 & <y<x \Longrightarrow\left(\frac{y}{x}\right)^{b_{\vee}-b_{\wedge}} \leq \frac{L_{1}^{\vee}(y)}{L_{1}^{\vee}(x)} \leq 1, \\
1 & \leq \frac{L_{1}^{\wedge}(y)}{L_{1}^{\wedge}(x)} \leq\left(\frac{y}{x}\right)^{b_{\wedge}-b_{\vee}}, \\
\left(\frac{y}{x}\right)^{b / 2} & \leq \frac{L_{2}(y)}{L_{2}(x)} \leq 2 .
\end{aligned}
$$

The following result is a consequence of Theorem 4 .
Corollary 2. Assume $\left(\mathbf{S}_{\mathbf{a}, \mathbf{b}}\right)$. Let $H_{i, t}$ be the p.d.f of $Y_{t}^{i}$, $i=1, N, t>0$. Let $T>0, T_{1}=[T, \infty), T_{N}=[0, T)$, and

$$
F_{i, t}^{k}(z)=\left|\frac{\left(H_{i, t}\right)^{(k)}(z)}{H_{i, t}(z)}\right|, \quad k \in \mathbb{N}, z \in \mathbb{R} .
$$

Then, for every nonnegative integer $s$, we have

$$
\begin{aligned}
& 0 \leq r \leq k \\
& 0<\rho<1-\frac{1}{\left(a_{1}+1\right)} \Longrightarrow \sup _{t \in T_{i}}\left(|z|^{r} F_{i, t}^{k}(z)\right)^{s} H_{i, t}(z)^{2(1-\rho)} \text { is integrable. }
\end{aligned}
$$

Proof. By Remark 5, notice that the Lévy measure of subordinator $Z$ in (99) has the required conditions. According to Theorem $4(\mathrm{c})$, these conditions imply that the family $\left(H_{1, t}\right)_{t \in T_{1}}$ behaves like $\left(G_{r}^{3}\right)_{r \in R_{3}}$ and that $\left(H_{N, t}\right)_{t \in T_{N}}$ behaves like $\left(G_{r}^{4}\right)_{r \in R_{4}}$ with $c=a_{1} / \alpha$ and $d=a_{N} / \alpha$.
Proof of Theorem 3. We will check the assumptions of Corollary 1 , which consist in the convergence of the integrals

$$
\begin{aligned}
I_{n} & =\int\left[1+z \frac{\left(H_{i, u_{n}}\right)^{\prime}}{H_{i, u_{n}}}(z)\right]^{2} H_{i, u_{n}}(z) \mathrm{d} z, \\
J_{n}(\rho) & =\left(\gamma u_{n}\right)^{(2 \rho-1) / \alpha} \int\left[\left|1+z \frac{\left(H_{i, u_{n}}\right)^{\prime}}{H_{i, u_{n}}}(z)\right|^{2}+\left|2+4 z \frac{\left(H_{i, u_{n}}\right)^{\prime}}{H_{i, u_{n}}}(z)+z^{2} \frac{\left(H_{i, u_{n}}\right)^{\prime \prime}}{H_{i, u_{n}}}(z)\right|\right]^{2} H_{i, u_{n}}(z)^{2(1-\rho)} \mathrm{d} z .
\end{aligned}
$$

The latter is guaranteed by Corollary 2, since the functions $F_{i, t}^{k}$ satisfy

$$
\sup _{n \in \mathbb{N}}\left(|z|^{r} F_{i, u_{n}}^{k}\right)^{s}\left(H_{i, u_{n}}\right)^{2(1-\rho)} \in L^{1}(\mathrm{~d} z),
$$

for $s \in\{0,1,2,3,4\}, r \in\{0,1, \ldots, k\}, 0 \leq k \leq 2$, and $\rho \leq 1-$ $\left(1 /\left(a_{1}+1\right)\right)$. The rest is obtained by reproducing the Proof of Theorem 2.

Remark 6. Notice that the main argument for proving Theorem 3 is the behavior of the densities uniformly in time. Theorem 4 provides many other examples. For example, with the same proof as in Theorem 3, one could state a version with stable processes time changed by any independent "nice" subordinator. The time change process could be the sum of a stable subordinator and a Poisson process (Remark 5). Finally, it appears that more investigation concerning the behavior in small time of p.d.f.'s of Lévy processes attracted by stable processes is crucial for statistical purposes.

\section{How to Build a LAN Model from Another LAN Model?}

In this section, we investigate to which extent the choice of the asymptotic is crucial. Assume we start from a LAN model associated to the observations of a Lévy process $X$ along a discretization scheme $i u_{n}, 1 \leq i \leq n$. Can we affirm that the model associated to the observations of $X+\widetilde{X}$, where $\widetilde{X}$ is another independent Lévy process, also enjoys the LAN property with the same discretization scheme? We need some preliminaries and two lemmas to answer the last question.

Consider two independent Lévy processes $Y$ and $N$ defined on some probability space $(\bar{\Omega}, \bar{F}, \overline{\mathbb{P}})$ with values in the Skorohod space $\Omega=\mathbb{D}(\mathbb{R}+, \mathbb{R})$ (when the processes $Y$ and $N$ are seen as infinite-dimensional random variables). Assume that $N$ is a nondrifted compound Poisson process with Lévy measure $v$, and consider the process $\widetilde{Y}:=Y+N$. Recall that the increment process $X^{n}$ of $X$, observed along a scheme $u_{n}$, is defined in (14) by 


$$
X_{j}^{n}=X_{(j+1) u_{n}}-X_{j u_{n}}, \quad 0 \leq j \leq n-1 .
$$
let

For $\theta \in \Theta$, suppose we observe $X^{n}=\theta Y^{n}, \widetilde{X}^{n}=\theta \widetilde{Y}^{n}$, and

$$
\begin{aligned}
& \mathbb{P}_{\theta}=\mathscr{L} a w(\theta Y \mid \overline{\mathbb{P}}), \\
& \widetilde{\mathbb{P}}_{\theta}=\mathscr{L} a w(\theta \widetilde{Y} \mid \overline{\mathbb{P}}) .
\end{aligned}
$$

The probability measure $\mathbb{P}_{\theta}^{n}$ (respectively, $\widetilde{\mathbb{P}}_{\theta}^{n}$ and the scale models $E^{n}$ (respectively, $\widetilde{E}^{n}$ ) correspond to $X$ (respectively, $\widetilde{X}$ ) as in (15) and (16).
Recall that if $Q, Q^{\prime}$ are two probability measures on some sample space, then the total variation distance $\left\|Q-Q^{\prime}\right\|$ is the quantity

$$
\begin{aligned}
\left\|Q-Q^{\prime}\right\| & =\sup _{\phi \in \Phi}\left|\mathbb{E}_{Q}(\phi)-\mathbb{E}_{Q^{\prime}}(\phi)\right|, \\
\Phi & =\{\phi: \Omega \longrightarrow[-1,1], \phi \text { measurable }\} .
\end{aligned}
$$

Lecam's lemma [2] is as follows:

Lemma 1. For every probability measures $Q, Q^{\prime}, R, R^{\prime}$, we have the inequality

$$
\int 1 \wedge\left|\frac{\mathrm{d} R}{\mathrm{~d} Q}-\frac{\mathrm{d} R^{\prime}}{\mathrm{d} Q^{\prime}}\right| \mathrm{d}\left(Q+Q^{\prime}\right) \leq\left\|Q-Q^{\prime}\right\|+2\left\|R-R^{\prime}\right\|+\left(2\left\|Q-Q^{\prime}\right\|\left\|R+R^{\prime}\right\|\right)^{1 / 2}
$$

We also have the following result.

Lemma 2. If $\lim _{n \longrightarrow \infty} n u_{n}=0$, then $\lim _{n \longrightarrow \infty} \sup _{\theta \in \Theta}$ $\left\|\mathbb{P}_{\theta}^{n}-\widetilde{\mathbb{P}}_{\theta}^{n}\right\|=0$.

Proof. Since

$$
\begin{aligned}
& \overline{\mathbb{P}}\left(N_{u_{n}} \in \mathrm{d} y\right)=e^{-\nu(\mathbb{R}) u_{n}} \sum_{k=0}^{\infty} \frac{u_{n}^{k}}{k !} \nu^{* k}(\mathrm{~d} y), \\
& \overline{\mathbb{P}}\left(N_{u_{n}}=0\right) \geq e^{-\nu(\mathbb{R}) u_{n}},
\end{aligned}
$$

and since $N$ has stationary and independent increments, we have

$$
\begin{aligned}
\left|\mathbb{P}_{\theta}^{n}-\widetilde{\mathbb{P}}_{\theta}^{n}\right| & =\sup _{\phi \in \Phi}\left|\mathbb{E}_{\mathbb{P}_{\theta}^{n}}[\phi(X)]-\mathbb{E}_{\tilde{\mathbb{P}}_{\theta}^{n}}[\phi(X)]\right|=\sup _{\phi \in \Phi}\left|\mathbb{E}_{\mathbb{P}_{\theta}}\left[\phi\left(X^{n}\right)\right]-\mathbb{E}_{\widetilde{\mathbb{P}}_{\theta}}\left[\phi\left(X^{n}\right)\right]\right| \\
& =\sup _{\phi \in \Phi}\left|\mathbb{E}_{\overline{\mathbb{P}}}\left[\phi\left(\theta Y^{n}\right)\right]-\mathbb{E}_{\overline{\mathbb{P}}}\left[\phi\left(\theta \widetilde{Y}^{n}\right)\right]\right|=\sup _{\phi \in \Phi}\left|\mathbb{E}_{\overline{\mathbb{P}}}\left[\phi\left(\theta Y^{n}\right)\right]-\mathbb{E}_{\overline{\mathbb{P}}}\left[\phi\left(\theta\left(Y^{n}+N^{n}\right)\right)\right]\right| \\
& =\sup _{\phi \in \Phi}\left|\mathbb{E}_{\overline{\mathbb{P}}}\left[\phi\left(\theta Y^{n}\right)\right]-\mathbb{E}_{\overline{\mathbb{P}}}\left[\phi\left(Y^{n}+N^{n}\right)\right]\right|=\sup _{\phi \in \Phi}\left|\mathbb{E}_{\overline{\mathbb{P}}}\left[\left(\phi\left(Y^{n}\right)-\phi\left(Y^{n}+N^{n}\right)\right) 1 l_{N^{n} \equiv / 0}\right]\right| \\
& \leq 2 \overline{\mathbb{P}}\left(N^{n} \neq 0\right)=2\left(1-\overline{\mathbb{P}}\left(N^{n} \equiv 0\right)\right)=2\left(1-\overline{\mathbb{P}}\left(N_{(j+1) u_{n}}-N_{j u_{n}}=0, \quad \forall 0 \leq j \leq n\right)\right) \\
& \leq 2\left(1-\overline{\mathbb{P}}\left(N_{u_{n}}=0\right)^{n}\right) \leq 2\left(1-e^{-\nu(\mathbb{R}) n u_{n}}\right) .
\end{aligned}
$$

It is now clear that $\left\|\mathbb{P}_{\theta}^{n}-\widetilde{\mathbb{P}}_{\theta}^{n}\right\|$ goes to 0 , uniformly in $\theta$ as $n u_{n} \longrightarrow 0$.

Now, we are able to complete Far's problem [18], which treats the case where $Y$ is a Brownian motion and where the discretization path is $u_{n}=1 / n$, i.e., $\lim _{n \longrightarrow \infty} n u_{n}=1$.

Theorem 5. Assume $\lim _{n \longrightarrow \infty} n u_{n}=0$. If the scale model (5) $E^{n}$ associated to the process $X$ has the LAN property with speed $\sqrt{n}$ in a point $\theta_{0} \in \Theta$, then so is the scale model $\widetilde{E}^{n}$ associated to the process $\widetilde{X}$.

Proof of Theorem 5. (1) Fix $\theta_{0} \in \Theta$, and $J$ is a finite subset of $\mathbb{R}$ and $\xi \in \mathbb{R}$. We shall prove that the weak functional convergence (22) of the likelihood processes $\left(Z^{n, \eta \xi}\right)_{\eta \in J}$ of $E^{n}$ yields the one of the likelihood processes $\left(\widetilde{Z}^{n, \eta \xi}\right)_{\eta \in J}$ of $\widetilde{E}_{n}$. The expression of the likelihood processes is given by (21), and for more convenience, we denote them from now on by

$$
\begin{aligned}
& \mathbf{Z}_{t}^{n}=\left(Z_{k}^{n, \eta \xi}\right)_{\eta \in J}, \\
& \widetilde{Z}_{t}^{n}=\left(\widetilde{Z}_{t}^{n, \eta \xi}\right)_{\eta \in J}, \\
& \mathbf{Z}_{t}^{\prime}=\left(Z_{t}^{\prime, \eta \xi}\right)_{\eta \in J}, \quad t \in[0,1] .
\end{aligned}
$$

We need to show the following convergence in laws:

$$
\mathscr{L} a w\left(Z^{n} \mid \mathbb{P}_{[\xi]_{n}}^{n}\right) \longrightarrow \mathscr{L} a w\left(\left(Z^{\prime, \eta \xi}\right)_{\eta \in J} \mid \mathbb{P}_{\xi}^{\prime}\right) \Longrightarrow \mathscr{L} a w\left(\widetilde{\mathbf{Z}}^{n} \mid \widetilde{\mathbb{P}}_{[\xi]_{n}}^{n}\right) \longrightarrow \mathscr{L} a w\left(\left(Z^{\prime, \eta \xi}\right)_{\eta \in J} \mid \mathbb{P}_{\xi}^{\prime}\right), \quad \text { as } n \longrightarrow \infty,
$$


or equivalently, for every $K$-Lipschitz function $f: \mathbb{D}\left(\mathbb{R}_{+}, \mathbb{R}^{J}\right) \longrightarrow \mathbb{R}$, bounded by a constant $C>0$, we need to show that

$$
\lim _{n \longrightarrow \infty} \mathbb{E}_{\mathbb{P}_{[\xi]_{n}}^{n}}\left[f\left(\mathbf{Z}^{n}\right)\right]=\mathbb{E}_{\mathbb{P}_{\xi}^{\prime}}\left[f\left(Z^{\prime}\right)\right] \Longrightarrow \lim _{n \longrightarrow \infty} \mathbb{E}_{\widetilde{\mathbb{P}}_{[\xi]_{n}}^{n}}\left[f\left(\widetilde{\mathbf{Z}}^{n}\right)\right]=\mathbb{E}_{\mathbb{P}_{\xi}^{\prime}}\left[f\left(Z^{\prime}\right)\right]
$$

(2) For such functions $f$, we will control the difference:

$$
\mathbb{E}_{\mathbb{P}_{[\xi]_{n}}^{n}}\left[f\left(\mathbf{Z}^{n}\right)\right]-\mathbb{E}_{\widetilde{\mathbb{P}}_{[\xi]_{n}^{n}}^{n}}\left[f\left(\widetilde{\mathbf{Z}}^{n}\right)\right]=\left(\mathbb{E}_{\mathbb{P}_{[\xi\}_{n}}^{n}}\left[f\left(\mathbf{Z}^{n}\right)\right]-\mathbb{E}_{\widetilde{\mathbb{P}}_{\left[\xi_{n}\right.}^{n}}\left[f\left(\mathbf{Z}^{n}\right)\right]\right)+\left(\mathbb{E}_{\widetilde{\mathbb{P}}_{[\xi]_{n}}^{n}}\left[f\left(\mathbf{Z}^{n}\right)\right]-\mathbb{E}_{\widetilde{\mathbb{P}}_{[\xi]_{n}}^{n}}\left[f\left(\widetilde{\mathbf{Z}}^{n}\right)\right]\right)
$$

In virtue of Lemma 2, we have

$$
\left|\mathbb{E}_{\mathbb{P}_{[\xi]_{n}}^{n}}\left[f\left(\mathbf{Z}^{n}\right)\right]-\mathbb{E}_{\widetilde{\mathbb{P}}_{\left[\xi_{n}\right]_{n}}^{n}}\left[f\left(\widetilde{\mathbf{Z}}^{n}\right)\right]\right| \leq C \sup _{\theta \in \Theta}\left\|\mathbb{P}_{\theta}^{n}-\widetilde{\mathbb{P}}_{\theta}^{n}\right\| \longrightarrow 0, \quad \text { as } n \longrightarrow \infty
$$

For every $\varepsilon>0$, we have

$$
\begin{aligned}
& \mathbb{E}_{\widetilde{\mathbb{P}}_{[\xi]_{n}}^{n}}\left[f\left(\mathbf{Z}^{n}\right)-f\left(\widetilde{\mathbf{Z}}^{n}\right)\right]=\mathbb{E}_{\widetilde{\mathbb{P}}_{[\xi]_{n}}^{n}}\left[\left(f\left(\mathbf{Z}^{n}\right)-f\left(\widetilde{\mathbf{Z}}^{n}\right)\right) 1 l_{\left.\left|\mathbf{Z}^{n}-\widetilde{\mathbf{Z}}_{\mid}^{n}\right|_{\leq \varepsilon}\right]+\mathbb{E}_{\widetilde{\mathbb{P}}_{[\xi]_{n}}^{n}}\left[\left(f\left(\mathbf{Z}^{n}\right)-f\left(\widetilde{\mathbf{Z}}^{n}\right)\right) 1 l_{\mid}\left|\mathbf{Z}^{n}-\widetilde{\mathbf{Z}}^{n}\right|>\varepsilon\right.}\right] \\
&\left|\mathbb{E}_{\widetilde{\mathbb{P}}_{[\xi]_{n}}^{n}}\left[f\left(\mathbf{Z}^{n}\right)\right]-f\left(\widetilde{\mathbf{Z}}^{n}\right)\right| \leq \varepsilon K+2 C \widetilde{\mathbb{P}}_{[\xi]_{n}}^{n}\left(\left|\mathbf{Z}^{n}-\widetilde{\mathbf{Z}}^{n}\right|>\varepsilon\right) .
\end{aligned}
$$

With representation (21), observe that $\mathbf{Z}^{n}$ and $\widetilde{\mathbf{Z}}^{n}$ are a step process, time-dependent, up to $\lfloor n t\rfloor, t \in[0,1]$. Then, denoting

$$
\tau^{n}=\inf \left\{1 \leq j \leq n \text { s.t. }\left|\mathbf{Z}_{j}^{n}-\widetilde{\mathbf{Z}}_{j}^{n}\right|>\varepsilon, \quad \forall \eta \in J, \xi \in \mathbb{R}\right\},
$$

$$
\begin{aligned}
\widetilde{\mathbb{P}}_{[\xi]_{n}}^{n}\left(\left|\mathbf{Z}^{n}-\widetilde{\mathbf{Z}}^{n}\right|>\varepsilon\right) & =\widetilde{\mathbb{P}}_{[\xi]_{n}}^{n}\left(\left|\mathbf{Z}_{\tau^{n}}^{n}-\widetilde{\mathbf{Z}}_{\tau^{n}}^{n}\right|>\varepsilon\right)=\widetilde{\mathbb{P}}_{[\xi]_{n}}^{n}\left(\left|\mathbf{Z}_{i}^{n}-\widetilde{\mathbf{Z}}_{j}^{n}\right|>\varepsilon, \quad \forall 1 \leq j \leq n\right) \\
& \leq \frac{1}{\varepsilon} \mathbb{E}_{\widetilde{\mathbb{P}}_{[\xi]_{n}}^{n}}\left[1 \wedge\left|\mathbf{Z}_{\tau^{n}}^{n}-\widetilde{\mathbf{Z}}_{\tau^{n}}^{n}\right|\right] .
\end{aligned}
$$

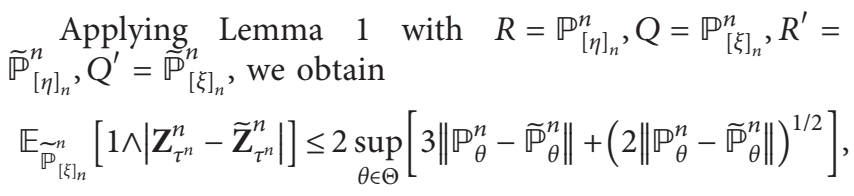

and then, Lemma 2 gives

$$
\lim _{n \longrightarrow \infty} \sup _{\eta \in J, \xi \in \mathbb{R}} \widetilde{\mathbb{P}}_{[\xi]_{n}}^{n}\left(\left|\mathbf{Z}^{n}-\widetilde{\mathbf{Z}}^{n}\right|>\varepsilon\right)=0 .
$$

The latter, together with (126), allows to conclude that

$$
\lim _{n \longrightarrow \infty} \mathbb{E}_{\mathbb{P}_{[\xi]_{n}}^{n}}\left[f\left(\mathbf{Z}^{n}\right)-f\left(\widetilde{\mathbf{Z}}^{n}\right)\right]=0 .
$$




\section{Data Availability}

No data were used to support this study.

\section{Conflicts of Interest}

The author declares that there are no conflicts of interest.

\section{Acknowledgments}

The work was supported by the "Research Supporting Project number (RSP-2021/162), King Saud University, Riyadh, Saudi Arabia." The author would like to thank the referees for their valuable comments and recommendations that improved the content of the article. The author is also grateful to Jean Jacod who introduced him to the topic of statistic of Lévy processes.

\section{References}

[1] O. E. Barndorff-Nielsen, T. Mikosch and S. I. Resnick, Lévy Processes: Theory and ApplicationsBirkhäuser, Boston, MA, USA, 2001.

[2] L. Lecam, Asymptotic Methods in Statistical Decision Theor$y$ Springer, New York, NY, USA, 1986.

[3] M. G. Akritas, "Asymptotic inference in Lévy processes of the dicontinuous type," Annals of Statistics, vol. 9, no. 3, pp. 604-614, 1981.

[4] H. Luschgy, "Local asymptotic mixed normality for semimartingale experiments," Probability Theory and Related Fields, vol. 92, no. 2, pp. 151-176, 1992.

[5] V. Genon-Catalot and J. Jacod, "Estimation of the diffusion coefficient for diffusion processes: random sampling," Scandinavian Journal of Statistics, vol. 21, no. 3, pp. 193-221, 1994.

[6] E. Clément and A. Gloter, "Local asymptotic mixed normality property for discretely observed stochastic differential equations driven by stable Lévy processes," Stochastic Processes and their Applications, vol. 125, no. 6, pp. 2316-2352, 2015.

[7] Y. Ait-Sahalia and J. Jacod, "Volatility estimators for discretely sampled Lévy processes," Annals of Statistics, vol. 35, no. 1, pp. 355-392, 2007.

[8] H. Masuda, "Joint estimation of discretely observed stable Lévy processes with symmetric Lévy density," Journal of the Japan Statistical Society, vol. 39, no. 1, pp. 49-75, 2009.

[9] R. Kawai and H. Masuda, "On the local asymptotic behavior of the likelihood function for Meixner Lévy processes under high-frequency sampling," Statistics and Probability Letters, vol. 81, no. 4, pp. 460-469, 2011.

[10] R. Kawai and H. Masuda, "Local asymptotic normality for normal inverse Gaussian Lévy processes with high-frequency sampling," ESAIM: Probability and Statistics, vol. 17, pp. 13-32, 2013.

[11] Y. Aït-Sahalia and J. Jacod, "Fisher's information for discretely sampled Lévy processes," Econometrica, vol. 76, no. 4, pp. 727-761, 2008.

[12] H. Rammeh, LPMA CNRS-UMR 7599, PhD thesis, University of Paris, Paris, France, 1994.

[13] J. Jacod and A. N. Shiryaev, Limit theorems for Stochastic ProcessesSpringer, Berlin Heidelberg, NY, USA, 1987.

[14] W. Jedidi, "Stable Processes, Mixing, and Distributional Properties. I," Theory of Probability and its Applications, vol. 52, no. 4, pp. 580-593, 2008.
[15] P. Protter, In Stochastic Integration and Differential Equations, Springer Verlag, Berlin, Heidelberg, Germany, 1990.

[16] V. M. Zolotarev, One Dimentional Stable LawsTransactions of the American Mathematical Society, Providence, RI, USA, 1986.

[17] W. Jedidi, "Stable Processes, Mixing, and Distributional Properties. II," Theory of Probability \& Its Applications, vol. 53, no. 1, pp. 81-105, 2009.

[18] H. Far, LPMA CNRS-UMR 7599,PhD thesis, Paris, France.

[19] H. Strasser, Mathematical Theory of Statistics, Statistical Experiment and Asymptotic Decision TheoryWalter de Gruyter., New York, NY, USA, 1985.

[20] J. Jacod, "Convergence of filtered statistical models and Hellinger processes," Stochastic Processes and their Applications, vol. 32, no. 1, pp. 47-68, 1989.

[21] J. Picard, "Density in small time for Lévy processes," ESAIM: Probability and Statistics, vol. 1, pp. 357-389, 1997.

[22] J. Bertoin and R. Doney, "Spitzer's condition for random walks and Lévy processes," Annales de l'Institut Henri Poincare (B) Probability and Statistics, vol. 33, no. 2, pp. 167-178, 1997. 